\title{
Thermal Radiation and MHD Effects on Free Convective Flow of a Polar Fluid through a Porous Medium in the Presence of Internal Heat Generation and Chemical Reaction
}

\author{
R. A. Mohamed, ${ }^{1}$ S. M. Abo-Dahab, ${ }^{1,2}$ and T. A. Nofal ${ }^{2}$ \\ ${ }^{1}$ Department of Mathematics, Faculty of Science, South Valley University, Qena 83523, Egypt \\ ${ }^{2}$ Department of Mathematics, Faculty of Science, Taif University, Taif, Saudi Arabia \\ Correspondence should be addressed to S. M. Abo-Dahab, sdahb@yahoo.com
}

Received 27 July 2010; Revised 20 October 2010; Accepted 19 November 2010

Academic Editor: Saad A. Ragab

Copyright (C) 2010 R. A. Mohamed et al. This is an open access article distributed under the Creative Commons Attribution License, which permits unrestricted use, distribution, and reproduction in any medium, provided the original work is properly cited.

\begin{abstract}
An analysis is presented to study the MHD free convection with thermal radiation and mass transfer of polar fluid through a porous medium occupying a semi-infinite region of the space bounded by an infinite vertical porous plate with constant suction velocity in the presence of chemical reaction, internal heat source, viscous and Darcy's dissipation. The highly nonlinear coupled differential equations governing the boundary layer flow, heat, and mass transfer are solved by using a two-term perturbation method with Eckert number $E$ as a perturbation parameter. The results are obtained for velocity, angular velocity, temperature, concentration, skin friction, Nusselt number, and Sherwood number. The effect of various material parameters on flow, heat, and mass transfer variables is discussed and illustrated graphically.
\end{abstract}

\section{Introduction}

Coupled heat and mass transfer problems in presence of chemical reaction are of importance in many processes and have, therefore, received considerable amount of attention in recent years. In processes such as drying, distribution of temperature and moisture over agricultural fields and graves of fruit trees, damage of crops due to freezing, evaporation at the surface of a water body, energy transfer in a wet cooling tower, and flow in a desert cooler, heat and mass transfer occur simultaneously. Possible applications of this type of flow can be found in many industries. For example, in the electric power industry, among the methods of generating electric power is one in which electrical energy is extracted directly from a moving conducting fluid. Chemical reactions can be modeled as either homogeneous or heterogeneous processes. This depends on whether they occur at an interface or as a single 
phase volume reaction. A homogeneous reaction is one that occurs uniformly throughout a given phase. The species generation in a homogeneous reaction is the same as internal source of heat generation. On the other hand, a heterogeneous reaction takes place in a restricted area or within the boundary of a phase. It can therefore be treated as a boundary condition similar to the constant heat flux condition in heat transfer. The study of heat and mass transfer with chemical reaction is of great practical importance to engineers and scientists because of its almost universal occurrence in many branches of science and engineering. Das et al. [1] have studied the effects of mass transfer on the flow past impulsively started infinite vertical plate with constant heat flux and chemical reaction. Diffusion of a chemically reactive species from a stretching sheet is studied by Anderson et al. [2]. Anjali Devi and Kandasamy [3] have analyzed the effects of chemical reaction, heat and mass transfer on laminar flow along a semi-infinite horizontal plate. Muthucumaraswamy and Ganesan [46] have studied the impulsive motion of a vertical plate with heat flux/mass flux/suction and diffusion of chemically reaction species. Muthucumaraswamy [7] has analyzed the effects of a chemical reaction on a moving isothermal vertical surface with suction. Ghaly and Seddeek [8] have discussed the Chebyshev finite difference method for the effects of chemical reaction, heat and mass transfer on laminar flow along a semi-infinite horizontal plate with temperature-dependent viscosity. Kandasamy et al. [9, 10] studied effects of chemical reaction, heat and mass transfer along a wedge with heat source and concentration in the presence of suction or injection, and chemical reaction, heat and mass transfer on MHD flow over a vertical stretching surface with heat source and thermal stratification effects. Mohamed et al. [11] have discussed the finite element method for the effect of a chemical reaction on hydromagnetic flow and heat transfer of a heat generation fluid over a surface embedded in a non-Darcian porous medium.

Convection problems associated with heat sources within fluid-saturated porous media are of great practical significance in a number of practical applications in geophysics and energy-related problems, such as recovery of petroleum resources, geophysical flow, cooling of underground electric cables, storage of nuclear waste materials, ground water pollution, fiber and granular insulations, solidification of costing, chemical catalytic reactors, and environmental impact of buried heat generating waste. Effect of heat generation or absorption on free convective flow with heat and mass transfer in geometries with and without porous media has been studied by many scientists and technologists [12-22].

The study of flow and heat transfer for an electrically conducting polar fluid past a porous plate under the influence of a magnetic field has attracted the interest of many investigators in view of its applications in many engineering problems such as magnetohydrodynamic (MHD) generator, plasma studies, nuclear reactors, oil exploration, geothermal energy extractions, and the boundary layer control in the field of aerodynamics [23]. Polar fluids are fluids with microstructure belonging to a class of fluids with nonsymmetrical stress tensor. Physically, the represented fluids are consisting of randomly oriented particles suspended in a viscous medium [24-26]. Ibrahim et al. [27] studied unsteady magnetohydrodynamic micropolar fluid flow and heat transfer over a vertical porous plate through a porous medium in the presence of thermal and mass diffusion with a constant heat source. Rahman and Sattar [28] studied MHD convective flow of a micropolar fluid past a vertical porous plate in the presence of heat generation/absorption. Kim [29] investigated MHD convection flow of polar fluids past a vertical moving porous plate in a porous medium. Helmy [30] obtained the solution for a magneto-hydromagnetic unsteady free convection flow past a vertical porous plate for a Newtonian fluid and a special type of non-Newtonion fluid known as micropolar fluids. Ogulu [31] studied the influence of 
radiation absorption on unsteady free convection and mass transfer flow of a polar fluid in the presence of uniform magnetic field. Anjali Devi and Kandasamy [32] have analyzed the effects of chemical reaction, heat and mass transfer on MHD flow past a semi infinite plate. The flow and mass transfer on a stretching sheet with a magnetic field and chemically reactive species are examined by Takhar et al. [33]. Raptis and Perdikis [34] have analyzed the effect of a chemical reaction of an electrically conducting viscous fluid on the flow over a nonlinearly (quadratic) semi-infinite stretching sheet in the presence of a constant magnetic field which is normal to the sheet. Seddeek [35] has studied the finite element for the effects of chemical reaction, variable viscosity, thermophoresis, and heat generation/absorption on a boundary layer hydromagnetic flow with heat and mass transfer over a heat source. Sharma and Thakur [36] have analyzed the effects of MHD on couple stress fluid heated from below in porous medium. V. Sharma and S. Sharma [37] have discussed effects of thermosolutal convection of micropolar fluids with MHD through a porous medium. The effect of heat and mass transfer on MHD micropolar flow over a vertical moving porous plate in a porous medium has studied by Kim [38]. The effect of rotation on a layer of micropolar ferromagnetic fluid heated from below saturating a porous medium is investigated by Sunil et al. [39].

Many processes are new engineering areas occuring at high temperatures, and knowledge of radiate heat transfer becomes very important for the design of the pertinent equipment. Nuclear power planets gas turbines and the various propulsion devices for aircraft, missiles, satellites, and space vehicles of radiation effects on the various types of flows are quite complicated. On the other hand, heat transfer by simultaneous free convection and thermal radiation in the case of a polar fluid has not received as much attention. This is unfortunate because thermal radiation plays an important role in determining the overall surface heat transfer in situations where convective heat transfer coefficients are small, as is the case in free convection such situations are common in space technology [40]. The effects of radiation on the flow and heat transfer of micropolar fluid past a continuously moving plate have been studied by many authors; see [41-44]. The radiation effect on heat transfer of a micropolar fluid past unmoving horizontal plate through a porous medium was studied by Abo-Eldahab and Ghonaim [45]. Ogulu [46] has studied the oscillating platetemperature flow of a polar fluid past a vertical porous plate in the presence of couple stresses and radiation. Rahman and Sultana [47] investigated the thermal radiation interaction of the boundary layer flow of micropolar fluid past a heated vertical porous plate embedded in a porous medium with variable suction as well as heat flux at the plate.

Recently, Mohamed and Abo-Dahab [48] investigated the effects of chemical reaction and thermal radiation on the heat and mass transfer in MHD micropolar flow over a vertical moving porous plate in a porous medium with heat generation.

The object of the present paper is to study the two-dimensional steady radiative heat and mass transfer flow of an incompressible, laminar, and electrically conductive viscous dissipative polar fluid flow through a porous medium, occupying a semi-infinite region of the space bounded by an infinite vertical porous plate in the presence of a uniform transverse magnetic field, chemical reaction of the first-order and internal heat generation. Approximate solutions to the coupled nonlinear equations governing the flow are derived and expression for the velocity, angular velocity, temperature, concentration, the rates of heat and mass transfer, and the skin-friction are derived. Numerical calculations are carried out; the purpose of the discussion of the results which are shown on graphs and the effects of the various dimensionless parameters entering into the problem on the velocity, angular velocity, temperature, concentration, the skin-friction, wall heat transfer, and mass transfer rates are studied. 


\section{Formulation of the Problem}

The basic equations of mass, linear momentum, angular momentum, energy and concentration for steady flow of polar fluids with the vector fields are as follows:

$$
\begin{aligned}
\nabla \cdot \mathbf{V}= & 0, \\
\rho(\mathbf{V} \cdot \nabla) \mathbf{V}= & \left(\mu+\mu_{r}\right) \nabla^{2} \mathbf{V}+2 \mu_{r}(\nabla \times \boldsymbol{\Omega})+\rho \mathbf{F}, \\
\rho k^{2}(\mathbf{V} \cdot \nabla) \boldsymbol{\Omega}= & \left(C_{a}+C_{d}\right)[\nabla(\nabla \cdot \boldsymbol{\Omega})-\nabla \times(\nabla \times \boldsymbol{\Omega})], \\
\rho c_{P}(\mathbf{V} \cdot \nabla) T^{*}= & \lambda \nabla^{2} T^{*}+\left(\mu+\mu_{r}\right)|\nabla \times \mathbf{V}|^{2}+\mu_{r} \Omega^{2}+2 \mu_{r}[\boldsymbol{\Omega} \cdot(\nabla \times \mathbf{V})] \\
& +\gamma^{*}|\nabla \times \boldsymbol{\Omega}|^{2}+\rho \mathbf{\Phi}-\nabla \cdot \mathbf{q}_{r}+Q, \\
(\mathbf{V} \cdot \nabla) C^{*}= & D^{*} \nabla^{2} C^{*}-K_{n} C^{n} .
\end{aligned}
$$

Here, $\mathbf{\Phi}$ is the dissipation function of mechanical energy per unit mass, $\mathbf{V}$ is the velocity vector, $\boldsymbol{\Omega}$ is the rotation vector, $\mathbf{F}$ is the body force vector, $\mu$ is the fluid viscosity, $\mu_{r}$ is the dynamic rotational viscosity, $n$ is the order of the reaction, $K_{n}$ is the rate constant, $Q=Q_{o}\left(T^{*}-\right.$ $\left.T_{\infty}^{*}\right)$ is the internal heat generation term, $\gamma^{*}$ is the spin-gradient of the fluid, and $\mathbf{q}_{r}$ is the heat flux vector included within the fluid as a result of temperature gradients.

We consider a two dimensional Carisian coordinates $\left(x^{*}, y^{*}\right)$, steady hydromagnetic free convection with thermal radiation and mass transfer flow of laminar, viscous, incompressible, and heat generation polar fluid through a porous medium occupying a semiinfinite region of the space bounded by an infinite vertical porous plate in the presence of a transverse magnetic field and chemical reaction. $x^{*}$ is taken along the vertical plate and $y^{*}$ is normal to it. The velocity, the angular velocity, the temperature, and the species concentration fields are $\left(u^{*}, v^{*}, 0\right),\left(0,0, w^{*}\right), T^{*}$, and $C^{*}$, respectively. The surface is maintained at a constant temperature $T_{w}^{*}$ different from the porous medium temperature $T_{\infty}^{*}$ sufficiently a way from the surface and allows a constant suction. The fluid is assumed to be a gray, emittingabsorbing, but nonscattering medium and the Rosseland approximation is used to describe the radiative heat flux in the energy equation. The radiative heat flux in the $x^{*}$-direction is considered negligible in comparison to the $y^{*}$-direction. A magnetic field of uniform strength is applied transversely to the direction of the flow. The magnetic Reynolds number of the flow is taken to be small enough so that the induced magnetic field can be neglected. No electric field is assumed to exit and both viscous and magnetic dissipations are neglected. A heat source is placed within the flow to allow possible heat generation effects. The concentration of diffusing species is assumed to be very small in comparison with other chemical species which are present; the concentration of species far from the surface $C_{\infty}^{*}$ is infinitesimally small [49] and hence the Soret and Duffer effects are neglected. However, the effects of the viscous dissipation and Darcy dissipation (ignoring the contribution due couple stresses as a first approximation) are accounted in the energy balance equation. The chemical reaction is taking place in the flow and all thermophysical properties are assumed to be constant. The flow is due to buoyancy effects arising from density variations caused by differences 
in the temperature as well as species concentration. The diagrammatic of the problem is displayed in Figure 1. The governing equations for this physical situation are based on the usual balance laws of mass, linear momentum, angular momentum, and energy and mass diffusion modified to account for the physical effects mentioned above.

These equations are given by

$$
\begin{gathered}
\frac{\partial v^{*}}{\partial y^{*}}=0 \\
v^{*} \frac{\partial u^{*}}{\partial y^{*}}=g \beta_{t}\left(T^{*}-T_{\infty}^{*}\right)+g \beta_{c}\left(C^{*}-C_{\infty}^{*}\right)+\left(v+v_{r}\right) \frac{\partial^{2} u^{*}}{\partial y^{* 2}} \\
+2 v_{r} \frac{\partial \omega^{*}}{\partial y^{*}}-\frac{v+v_{r}}{K^{\prime}} u^{*}-\frac{\sigma B_{o}^{2}}{\rho} u^{*}, \\
v^{*} \frac{\partial \omega^{*}}{\partial y^{*}}=\frac{\gamma}{I} \frac{\partial^{2} \omega^{*}}{\partial y^{* 2}}, \\
v^{*} \frac{\partial T^{*}}{\partial y^{*}}=\frac{\lambda}{\rho c_{P}} \frac{\partial^{2} T^{*}}{\partial y^{* 2}}+\frac{Q_{o}}{\rho c_{P}}\left(T^{*}-T_{\infty}^{*}\right) \\
+\frac{v}{c_{P}}\left(\frac{\partial u^{*}}{\partial y^{*}}\right)^{2}+\frac{v}{K^{\prime} c_{P}} u^{* 2}-\frac{1}{\rho c_{P}} \frac{\partial q_{r}^{*}}{\partial y^{*}} \\
v^{*} \frac{\partial C^{*}}{\partial y^{*}}=D^{*} \frac{\partial^{2} C^{*}}{\partial y^{* 2}}-K_{1} C^{*}
\end{gathered}
$$

after employing the "volume averaging" process [50] and $\gamma=\left(C_{a}+C_{d}\right)$.

Since the fluid is viscous and especially a fluid with couple stress, according to following D'ep [25], the boundary conditions are

$$
\begin{aligned}
& u^{*}=0, \quad T^{*}=T_{w}^{*}, \quad C^{*}=C_{w}^{*}, \quad \frac{\partial w^{*}}{\partial y^{*}}=-\frac{\partial^{2} u^{*}}{\partial y^{*}}, \quad \text { at } y^{*}=0, \\
& u^{*} \longrightarrow \infty, \quad w^{*} \longrightarrow 0, \quad T^{*} \longrightarrow T_{\infty}^{*}, \quad C^{*} \longrightarrow C_{\infty}^{*}, \quad \text { as } y^{*} \longrightarrow \infty
\end{aligned}
$$

These boundary condition are derived from the assumption that the couple stresses are dominant during the rotation of the particles.

The radiative heat flux under Rosseland approximation (see, Raptis [41], Sparrow and Cess [51]), $q_{r}^{*}$, takes the form

$$
q_{r}^{*}=-\frac{4 \sigma^{*}}{3 K^{*}} \frac{\partial T^{* 4}}{\partial y^{*}}
$$




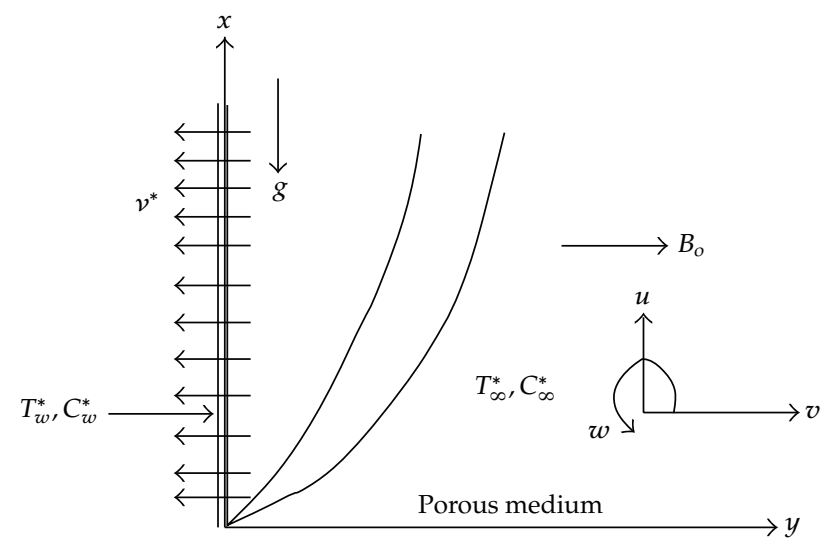

Figure 1: Schematic of the problem.

It should be noted that by using the Rosseland approximation, we limit our analysis to optically thick boundary layer and assuming the Eckert number to be small $E \ll 1$.

If the temperature differences within the flow are sufficiently small, then (2.7) can be generalized by expanding $T^{* 4}$ into the Taylor's series about $T_{\infty}^{*}$ and neglecting higher-order terms, which gives [41]

$$
T^{*} \cong T_{\infty}^{* 4}+4\left(T^{*}-T_{\infty}^{*}\right) T_{\infty}^{* 3}=4 T_{\infty}^{* 3} T^{*}-3 T_{\infty}^{* 4}
$$

The works [41-48] and many authors used the linearized from (2.8) of thermal radiations. Linearized thermal radiation, (2.8), makes the problem easy to handle; in this work we use linear form of thermal radiation.

By using (2.7) and (2.8) into (2.4), we get

$$
v^{*} \frac{\partial T^{*}}{\partial y^{*}}=\frac{\lambda}{\rho c_{P}} \frac{\partial^{2} T^{*}}{\partial y^{* 2}}+\frac{Q_{o}}{\rho c_{P}}\left(T^{*}-T_{\infty}^{*}\right)+\frac{v}{c_{P}}\left(\frac{\partial u^{*}}{\partial y^{*}}\right)^{2}+\frac{v}{K^{\prime} c_{P}} u^{* 2}+\frac{16 \sigma^{*}}{3 \rho c_{P}} \frac{T^{* 3}}{K^{*}} \frac{\partial^{2} T^{*}}{\partial y^{* 2}}
$$

The integration of the continuity (2.2) yields

$$
v^{*}=-v_{0}
$$

where $v_{o}$ is the constant suction velocity at the wall and the negative sign indicates that the suction velocity is directed towards the plate.

We Introduce the following nondimensional quantities:

$$
y=\frac{y^{*} v_{o}}{v}, \quad u=\frac{u^{*}}{v_{o}}, \quad \theta=\frac{T^{*}-T_{\infty}^{*}}{T_{w}^{*}-T_{\infty}^{*}}, \quad \omega=\frac{\omega^{*} v}{v_{o}^{2}}, \quad \phi=\frac{C^{*}-C_{\infty}^{*}}{C_{w}^{*}-C_{\infty}^{*}} .
$$



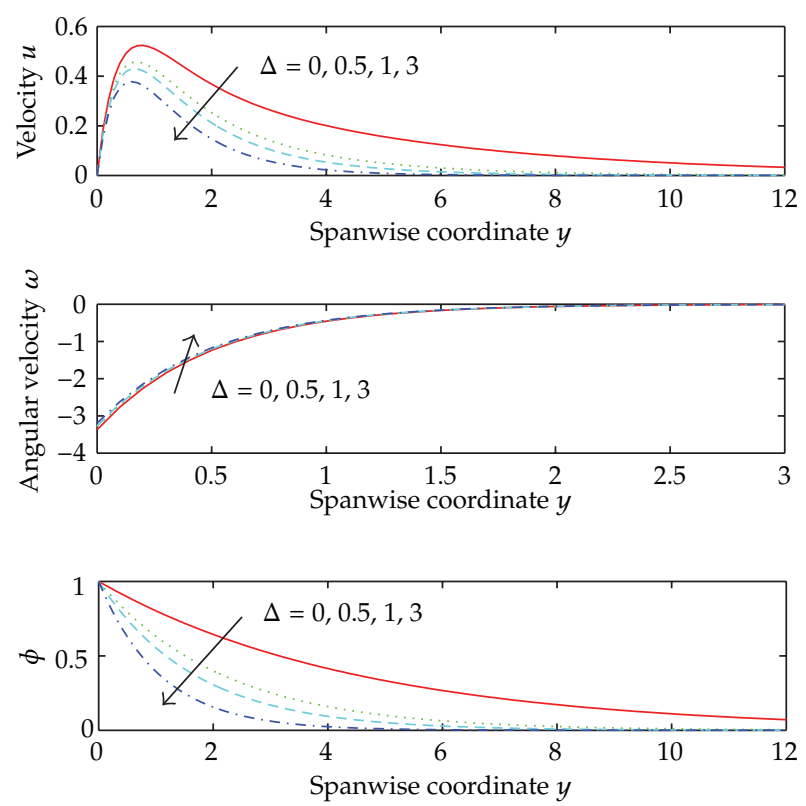

Figure 2: Effect of $\Delta$ on $u, \omega$ and $\phi$ respect to $y$, where, $K=0.5, \operatorname{Pr}=0.71, Q=0.5, \alpha=R=0.1, S c=0.22$, $\mathrm{Gm}=\mathrm{Gr}=M=2, E=0.01$, and $\beta=2$.

Substituting from (2.11) into (2.3), (2.5), and (2.9) and taking into account (2.10), we obtain

$$
\begin{aligned}
(1+\alpha) u^{\prime \prime}+u^{\prime}-\left(\frac{1+\alpha+K M}{K}\right) u & =-\left(G r \theta+G m \phi+2 \alpha \omega^{\prime}\right), \\
\omega^{\prime \prime}+\beta \omega^{\prime} & =0, \\
\left(1+\frac{4 R}{3}\right) \theta^{\prime \prime}+\operatorname{Pr} \theta^{\prime}+\operatorname{Pr} Q \theta & =-\operatorname{Pr} E\left(u^{\prime 2}+\frac{u^{2}}{K}\right), \\
\phi^{\prime \prime}+S c \phi^{\prime}-\Delta S c \phi & =0 .
\end{aligned}
$$

The mass diffusion (2.13) can be adjusted to represent a destructive reaction if $\Delta>0$, no chemical reaction if $\Delta=0$ and generation reaction if $\Delta<0$.

The dimensionless form of the boundary conditions (2.6) becomes

$$
\begin{aligned}
& u=0, \quad \omega^{\prime}=-u^{\prime \prime}, \quad \theta=1, \quad \phi=1, \quad \text { at } y=0, \\
& u \longrightarrow 0, \quad \omega \longrightarrow 0, \quad \theta \longrightarrow 0, \quad \phi \longrightarrow 0, \quad \text { as } y \longrightarrow \infty,
\end{aligned}
$$



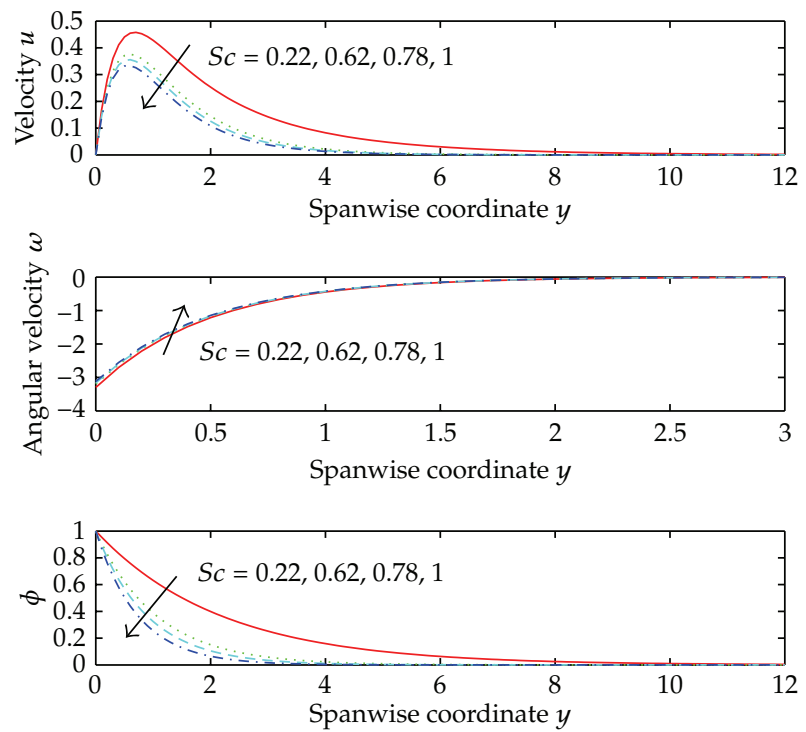

Figure 3: Effect of $S c$ on $u, \omega$ and $\phi$ respect to $y$, where, $K=0.5, \operatorname{Pr}=0.71, Q=0.5, \alpha=R=0.1, \Delta=0.5$, $G m=G r=M=2, E=0.01$, and $\beta=2$.

where prime denotes the differentiation with respect to $y$, and

$$
\begin{gathered}
\alpha=\frac{v_{r}}{v}, \quad \beta=\frac{I v}{\gamma}, \quad \operatorname{Pr}=\frac{\rho v c_{P}}{\lambda}, \quad E=\frac{v_{o}^{2}}{c_{P}\left(T_{w}^{*}-T_{\infty}^{*}\right)}, \quad R=\frac{4 \sigma^{*} T_{\infty}^{*}}{K^{*} \lambda}, \\
G r=\frac{v g \beta_{t}\left(T_{w}^{*}-T_{\infty}^{*}\right)}{v_{o}^{3}}, \quad G m=\frac{v g \beta_{c}\left(C_{w}^{*}-C_{\infty}^{*}\right)}{v_{o}^{3}}, \quad K=\frac{K^{\prime} v_{o}^{2}}{v^{2}}, \\
Q=\frac{Q_{o} v}{\rho c_{P} v_{o}^{2}}, \quad S c=\frac{v}{D^{*}}, \quad \Delta=\frac{K_{1} v}{v_{o}^{2}}, \quad M=\frac{\sigma B_{o}^{2} v}{\rho v_{o}^{2}}
\end{gathered}
$$

where the variables and related quantities are defined in the nomenclature.

The problem under consideration is now reduced to the system of (2.12)-(2.14), the solutions of which are obtained in the following section.

\section{Solution of the Problem}

The exact solution of (2.13) subject to the corresponding boundary conditions (2.14) takes the form

$$
\phi(y)=\operatorname{Exp}\left(R_{1} y\right),
$$



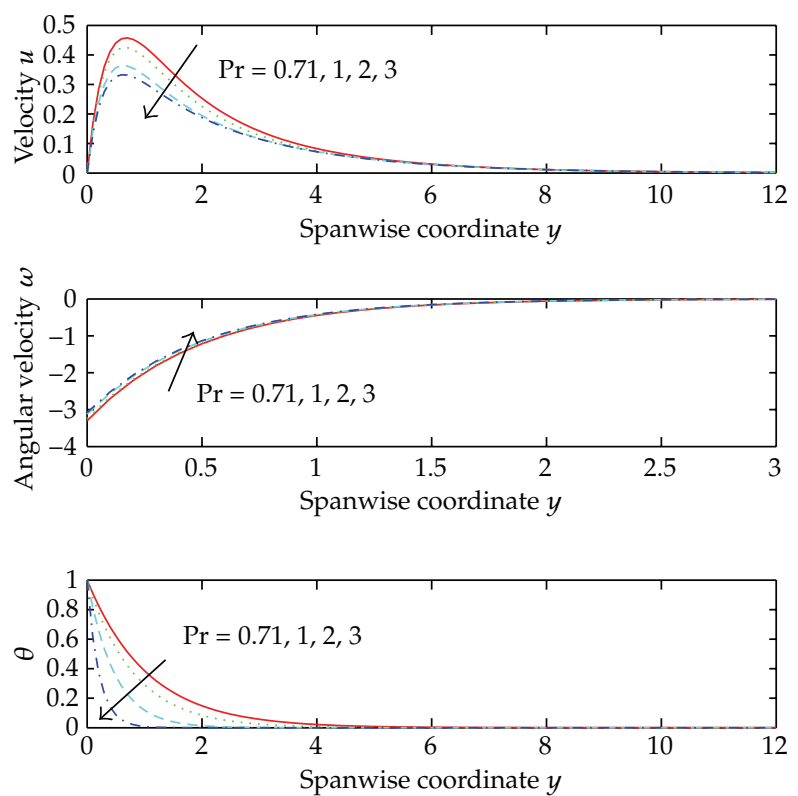

Figure 4: Effect of $\operatorname{Pr}$ on $u, \omega$, and $\theta$ respect to $y$, where, $K=0.5, S c=0.22, Q=0.5, \alpha=R=0.1, \Delta=0.5$, $G m=G r=M=2, E=0.01$, and $\beta=2$.
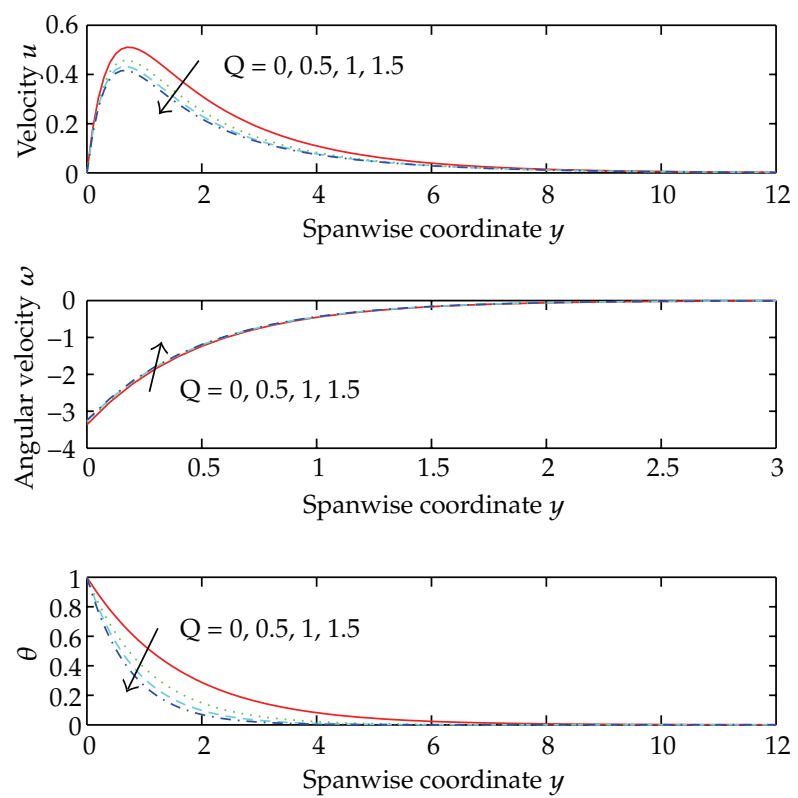

Figure 5: Effect of $Q$ on $u, \omega$, and $\theta$ respect to $y$, where, $K=0.5, \operatorname{Pr}=0.71, S c=0.22, \alpha=R=0.1, \Delta=0.5$, $G m=G r=M=2, E=0.01$, and $\beta=2$. 

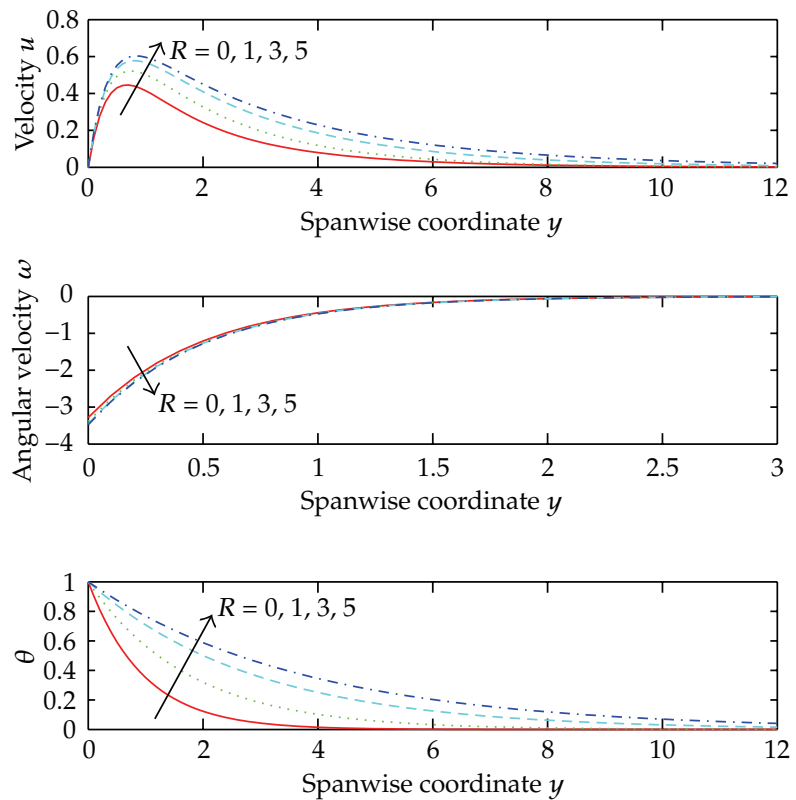

Figure 6: Effect of $R$ on $u, \omega$, and $\theta$ respect to $y$, where, $K=0.5$, $\operatorname{Pr}=0.71, Q=0.5, \alpha=S c=0.22, \Delta=0.5$, $\mathrm{Gm}=\mathrm{Gr}=M=2, E=0.01$, and $\beta=2$.

where

$$
R_{1}=\frac{-S c-\sqrt{S c^{2}+4 S c \Delta}}{2}
$$

The problem posed in (2.12) subject to the boundary condition presented in (2.14) is highly non-linear coupled equations and generally will involve a step by step numerical integration of the explicit finite difference scheme. However, analytical solutions are possible. Since viscous dissipation parameter $E$ is very small in most of the practical problems, therefore, we can advance an asymptotic expansion with $E$ as perturbation parametric for the velocity, angular velocity, and temperature profile as follow:

$$
\begin{aligned}
& u=u_{0}(y)+E u_{1}(y)+O\left(E^{2}\right), \\
& \omega=\omega_{0}(y)+E \omega_{1}(y)+O\left(E^{2}\right), \\
& \theta=\theta_{0}(y)+E \theta_{1}(y)+O\left(E^{2}\right),
\end{aligned}
$$

where the zeroth order terms correspond to the case in which the viscous and Darcy's dissipation is neglected $(E=0)$. By the substitution of (3.3) into (2.12) and the boundary conditions (2.14), we get the following system of equations. 

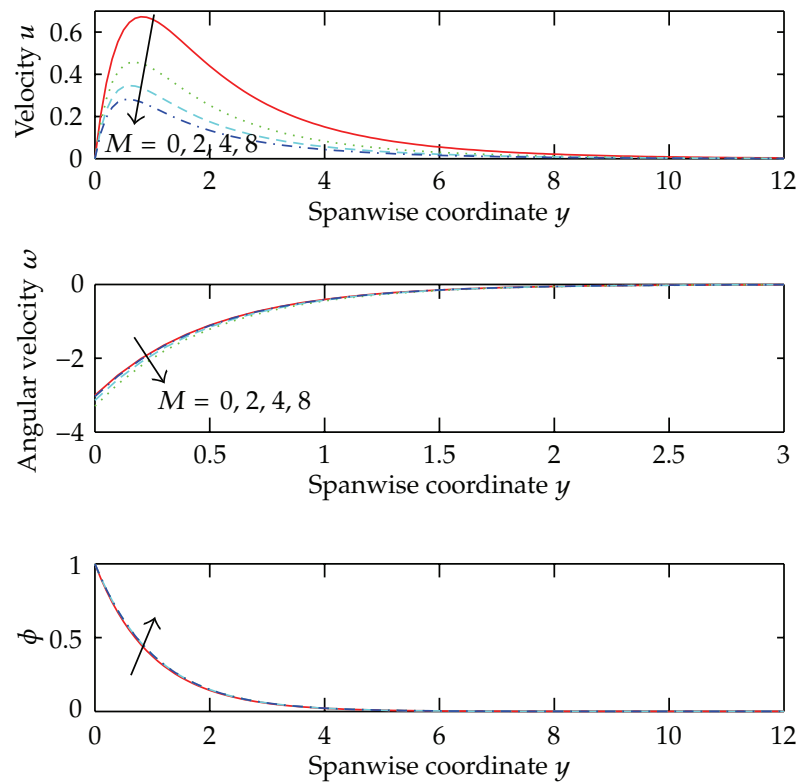

Figure 7: Effect of $M$ on $u, \omega$, and $\theta$ respect to $y$, where, $K=0.5, S c=0.22, \Delta=0.5, \operatorname{Pr}=0.71, Q=0.5$, $\alpha=R=0.1, G m=G r=2, E=0.01$, and $\beta=2$.

Zeroth order

$$
\begin{gathered}
(1+\alpha) u_{0}^{\prime \prime}+u_{0}^{\prime}-\left(\frac{1+\alpha+K M}{K}\right) u_{0}=-\left(G r \theta_{0}+G m \phi+2 \alpha \omega_{0}^{\prime}\right), \\
\omega_{0}^{\prime \prime}+\beta \omega_{0}^{\prime}=0 \\
\left(1+\frac{4 R}{3}\right) \theta_{0}^{\prime \prime}+\operatorname{Pr}\left(\theta_{0}^{\prime}+Q \theta_{0}\right)=0
\end{gathered}
$$

subject to the reduced boundary conditions

$$
\begin{aligned}
& u_{o}=0, \quad \omega_{0}^{\prime}=-u_{0}^{\prime \prime}, \quad \theta_{0}=1, \quad \text { at } y=0, \\
& u_{0} \longrightarrow 1, \quad \omega_{0} \longrightarrow 0, \quad \theta_{0} \longrightarrow 0, \quad \text { as } y \longrightarrow \infty
\end{aligned}
$$

First order

$$
\begin{gathered}
(1+\alpha) u_{1}^{\prime \prime}+u_{1}^{\prime}-\left(\frac{1+\alpha+K M}{K}\right) u_{1}=-\left(G r \theta_{1}+2 \alpha \omega_{1}^{\prime}\right), \\
\omega_{1}^{\prime \prime}+\beta \omega_{1}^{\prime}=0 \\
\left(1+\frac{4 R}{3}\right) \theta_{1}^{\prime \prime}+\operatorname{Pr}\left(\theta_{1}^{\prime}+Q \theta_{1}\right)=-\operatorname{Pr}\left(u_{0}^{\prime 2}+\frac{u_{0}^{2}}{K}\right)
\end{gathered}
$$



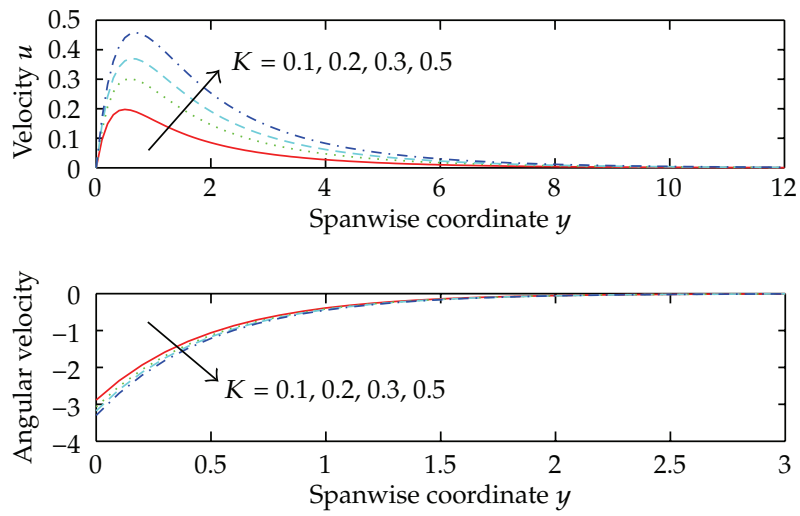

Figure 8: Effect of $K$ on $u$ and $\omega$ respect to $y$, where, $M=2, S c=0.22, \Delta=0.5, \operatorname{Pr}=0.71, Q=0.5$, $\alpha=R=0.1, G m=G r=2, E=0.01$, and $\beta=2$.
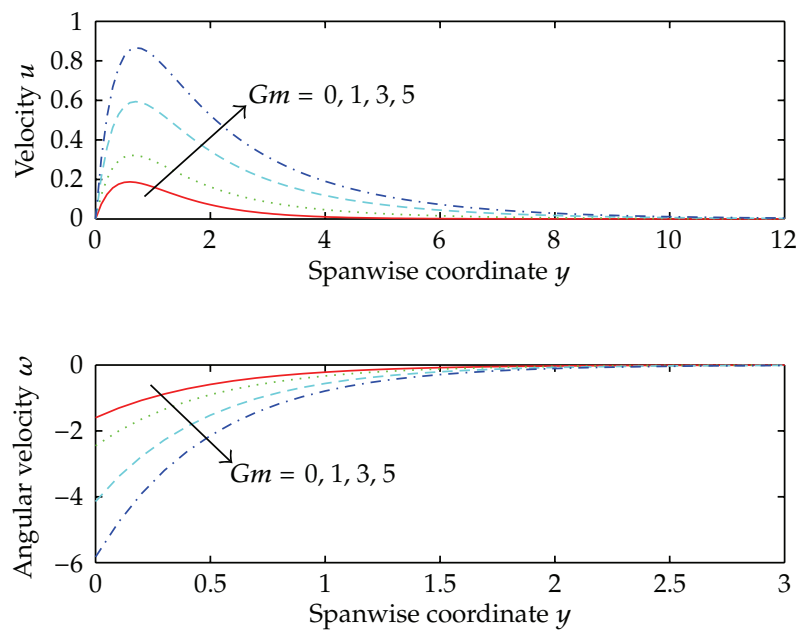

Figure 9: Effect of $G m$ on $u$ and $\omega$ respect to $y$, where, $M=2, S c=0.22, \Delta=0.5, \operatorname{Pr}=0.71, Q=0.5$, $\alpha=R=0.1, D=0.5, G r=2, E=0.01$, and $\beta=2$.

subject to the reduced boundary conditions

$$
\begin{aligned}
& u_{1}=0, \quad \omega_{1}^{\prime}=-u_{1}^{\prime \prime}, \quad \theta_{1}=0, \quad \text { at } y=0, \\
& u_{1} \longrightarrow 0, \quad \omega_{0}^{\prime} \longrightarrow 0, \quad \theta_{1} \longrightarrow 0, \quad \text { as } y \longrightarrow \infty .
\end{aligned}
$$



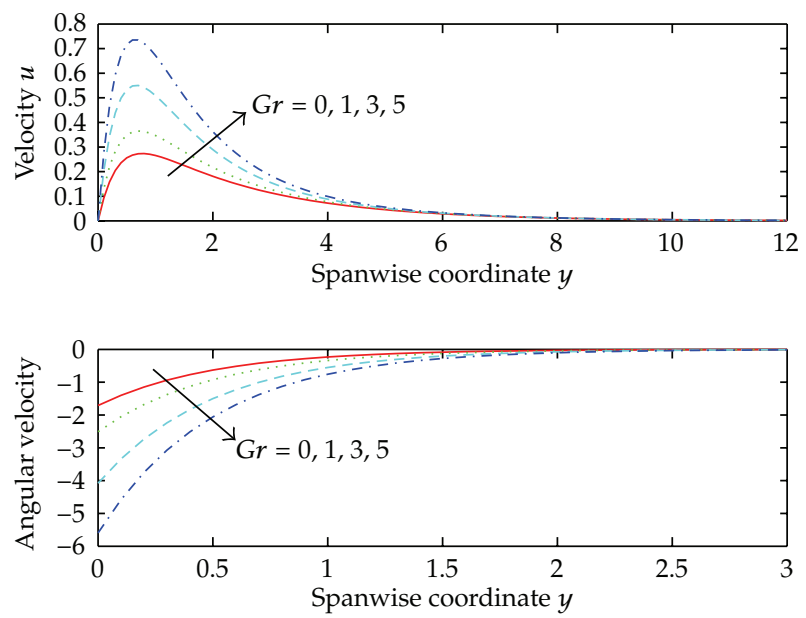

Figure 10: Effect of $G r$ on $u$ and $\omega$ respect to $y$, where, $M=2, S c=0.22, \Delta=0.5, \operatorname{Pr}=0.71, Q=0.5$, $\alpha=R=0.1, D=0.5, G m=2, E=0.01$, and $\beta=2$.

Solving (3.4) under the boundary conditions in (3.5) and (3.6) under the boundary conditions (3.7) and substituting the solutions into (3.3), we obtain

$$
\begin{gathered}
u(y)=C_{2} e^{R_{5} y}+A_{1} e^{R_{3} y}+A_{2} e^{R_{1} y}+A_{3} C_{1} e^{-\beta y} \\
+E\left[C_{4} e^{R_{5} y}+A_{14} e^{R_{3} y}+A_{15} e^{2 R_{5} y}+A_{16} e^{2 R_{3} y}+A_{17} e^{2 R_{1} y}+A_{18} e^{2 \beta y}\right. \\
+A_{19} e^{\left(R_{3}+R_{5}\right) y}+A_{20} e^{\left(R_{1}+R_{5}\right) y}+A_{21} e^{\left(R_{5}-\beta\right) y}+A_{22} e^{\left(R_{1}+R_{3}\right) y} \\
\left.+A_{23} e^{\left(R_{3}-\beta\right) y}+A_{24} e^{\left(R_{1}-\beta\right) y}+A_{25} C_{3} e^{-\beta y}\right]+O\left(E^{2}\right) \\
\begin{aligned}
& w(y)=\left[C_{1}+E C_{3}\right] e^{-\beta y}+O\left(E^{2}\right) \\
& \theta(y)=e^{R_{3} y}+ E\left[D_{1} e^{R_{3} y}+A_{4} e^{2 R_{5} y}+A_{5} e^{2 R_{3} y}+A_{6} e^{2 R_{1} y}\right. \\
&+A_{7} e^{-2 \beta y}+A_{8} e^{\left(R_{3}+R_{5}\right) y}+A_{9} e^{\left(R_{1}+R_{5}\right) y}+A_{10} e^{\left(R_{5}-\beta\right) y} \\
&\left.+A_{11} e^{\left(R_{1}+R_{3}\right) y}+A_{12} e^{\left(R_{3}-\beta\right) y}+A_{13} e^{\left(R_{1}-\beta\right) y}\right]+O\left(E^{2}\right)
\end{aligned}
\end{gathered}
$$

where the exponential indices and coefficients are given in the Appendix. 

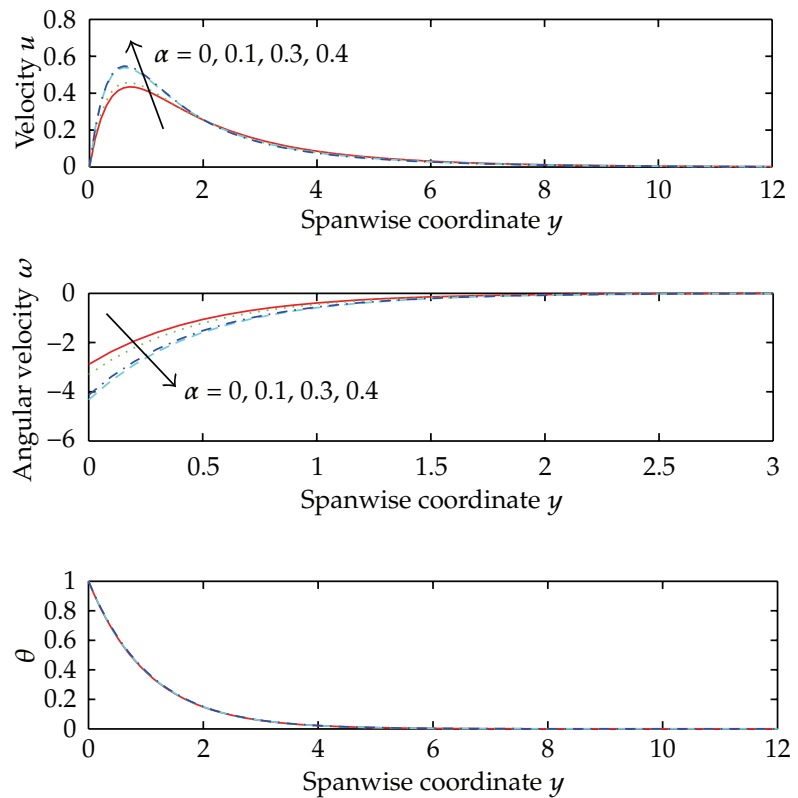

Figure 11: Effect of $\alpha$ on $u, w$, and $\theta$ respect to $y$, where, $M=2, S c=0.22, \Delta=0.5, \operatorname{Pr}=0.71, Q=0.5$, $R=0.1, D=0.5, G m=G r=2, E=0.01$, and $\beta=2$.

From the engineering point of view, the most important characteristics of the flow are the skin friction coefficient $C_{f}$, Nuselt $N u$, and Scherwood $S h$ numbers, which are given below:

$$
\begin{gathered}
C_{f}=\left(\frac{d u}{d y}\right)_{y=0}=\left(\frac{d u_{0}}{d y}+E \frac{d u_{1}}{d y}\right)_{y=0}, \\
N u=-\left(1+\frac{4 R}{3}\right)\left(\frac{d \theta}{d y}\right)_{y=0}=-\left(1+\frac{4 R}{3}\right)\left(\frac{d \theta_{0}}{d y}+E \frac{d \theta_{1}}{d y}\right)_{y=0}, \\
S h=-\left(\frac{\partial \phi}{\partial y}\right)_{y=0}=-R_{1} .
\end{gathered}
$$

If the thermal radiation and uniform transverse magnetic field are neglected, all the relevant results obtained are deduced to the results obtained in [22].

\section{Numerical Results and Discussions}

For a computation work, we used a Matlab program as a software, to illustrate the behavior of velocity $u$, angular velocity $\omega$, temperature $\theta$, and concentration $\phi$ fields; a numerical computation is carried out for various values of the parameters that describe the flow characteristics and the results are reported in terms of graphs. This is done in order to 

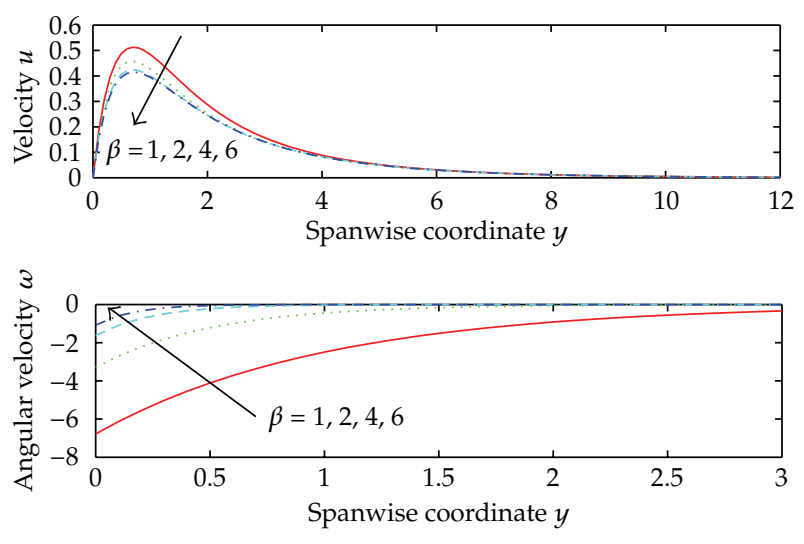

Figure 12: Effect of $\beta$ on $u$ and $\omega$ respect to $y$, where, $M=2, S c=0.22, \Delta=0.5, \operatorname{Pr}=0.71, Q=0.5$, $R=0.1, D=0.5, G m=G r=2, E=0.01$, and $\alpha=0.1$.

illustrate the special features of the solutions. The chemical reaction parameter $\Delta=0,0.5,1,3$, where $\Delta=0$ corresponds to the case of no chemical reaction. Figure 2 presents the profiles of the velocity, angular velocity, and concentration for various values of chemical reaction parameter $\Delta>0$. It is noted from Figure 2 that an increase in the values of chemical reaction parameter leads to decrease in the velocity and concentration and an increase in angular velocity of the polar fluid. The MHD and the concentration boundary layer become thin as the reaction parameters. The negative values of the angular velocity indicate that the angular velocity of substructures in the polar fluid is clockwise. Schmidt number $S c$ is chosen for hydrogen $(S c=0.22)$, water-vapor $(S c=0.62)$, and ammonia $(S c=0.78)$ at temperature $25^{\circ} \mathrm{C}$ and one atmospheric pressure. It is noted from Figure 3 that an increasing of the values of Schmidt number $S c$ leads a decrease in velocity and concentration. Physically, the increase of $S c$ means the decrease of molecular diffusivity $D^{*}$. That results in a decrease of concentration boundary layer, hence, the concentration of the spices for small values of $S c$ and lower for larger values of Sc. Also, it appears that there is a slight change in angular velocity with various values of $S c$.

The values of Prandtl number Pr are chosen to be $\operatorname{Pr}=0.71,1,2,3$. The effect of buoyancy is significant for $\operatorname{Pr}=0.71$ (air) due to the lower density. Figure 4 displays the effect of the Prandtl number Pr on the velocity, angular velocity, and temperature; it is clear that an increase of Pr leads to a decrease in $u$ that physically is true because the increase in the Prandtl number is due to the increase in the viscosities of the fluid which makes the fluid thick and hence causes a decrease in the velocity of fluid; the temperature $\theta$ decreases with the increasing of Pr; clearly, the increase of Prandtl number leads to a decreasing thermal boundary layer thickness and more uniform temperature distribution across the boundary layer. This results explain the fact that smaller values of Pr are equivalent to increasing the thermal conductivities, so that heat is able to diffuse away from the surface more rapidly than for higher values of Pr. Therefore, the boundary layer becomes thicker which finally reduces the temperature, but slight change in angular velocity $\omega$ with causes the increasing of Pr. The internal heat generation parameter $Q$ is chosen to be $Q=0,0.5,1,1.5$, where $Q=0$ corresponds to the case of no heat source. The effects of internal heat generation parameter $Q$ 

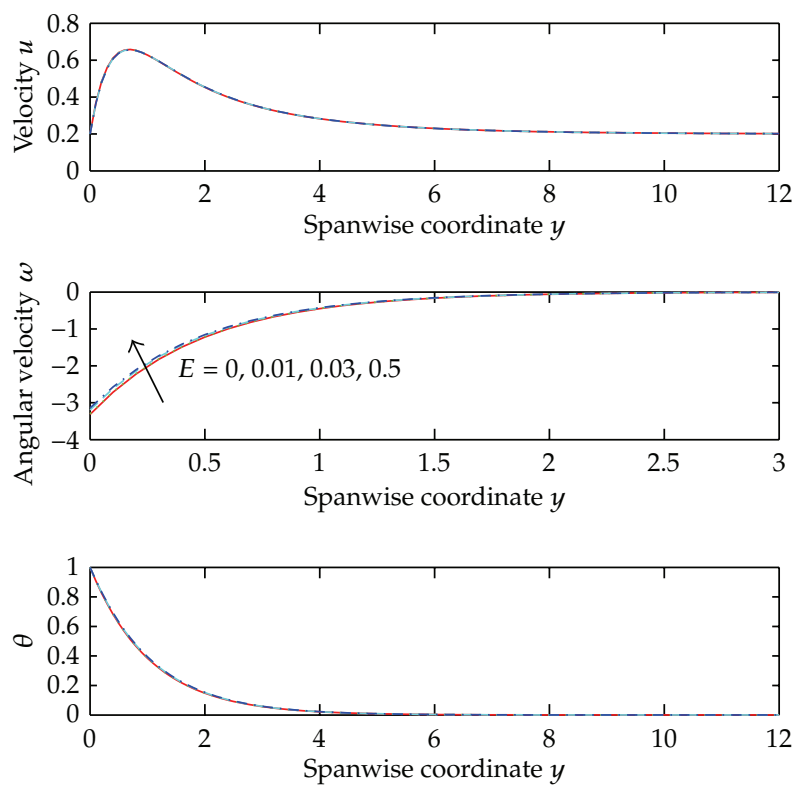

Figure 13: Effect of $E$ on $u, \omega$, and $\theta$ respect to $y$, where, $M=2, S c=0.22, \Delta=0.5, \operatorname{Pr}=0.71, Q=0.5$, $R=0.1, D=0.5, G m=G r=2, \beta=2$, and $\alpha=0.1$.

on the velocity and angular velocity are displayed in Figure 5. It is clear that as the parameter $Q$ increases, the velocity and angular velocity (in magnitude) lead to a fall. Further, it is noticed that the temperature decreases; this result qualitatively agrees with the results in [22].

The radiation parameter $R$ is chosen to be $R=0,1,3,5$, where $R=0$ corresponds to the case of no thermal radiation. The effects of radiation parameter $R$ on the velocity and angular velocity are displayed in Figure 6; it is clear that as the parameter $R$ increases, the velocity and temperature increase. Also, it is noticed that the angular velocity decreases. In the aiding flow, the effect of thermal radiation is to (i) increase the convection moment in the boundary layer, (ii) increase the thermal boundary-layer thickness with an increase in the value of the radiation parameter, and (iii) enhance the heat transfer coefficient in the medium. The effect of magnetic field $M$ parameter is shown in Figure 7, $M=0,2,4,6$, where, $M=0$ indicates to neglectes the magnetic field; it is observed that an increasing of the magnetic field leads to decreasing of $u$ because the application of transverse magnetic field will result in a restrictivetype force (Lorenz's force) similar to drag force which tends to resist the fluid flow and thus reducing its velocity; also, it appears that increasing of $M$ tends to increasing of $\omega$ and a slight change in $\theta$. The effect of permeability $K$ parameter is shown in Figure $8, K=0.1,0.2,0.3,0.5$; it is observed that an increase in the permeability leads to an increase of $u$ but a decrease of $\omega$. This is due to the fact that the presence of a porous medium increases the resistance to flow and when $K$ tends to infinity (i.e., the porous media effects vanish), the velocity is greater in the flow fluid. These results similar to those of Patil and Kulharni [22] could be very useful in deciding the applicability of enhanced oil recovery in reservoir engineering. The effects of solutal Grashof number $G m$ and Grashof number $G r$ on $u$ and wrespect to 

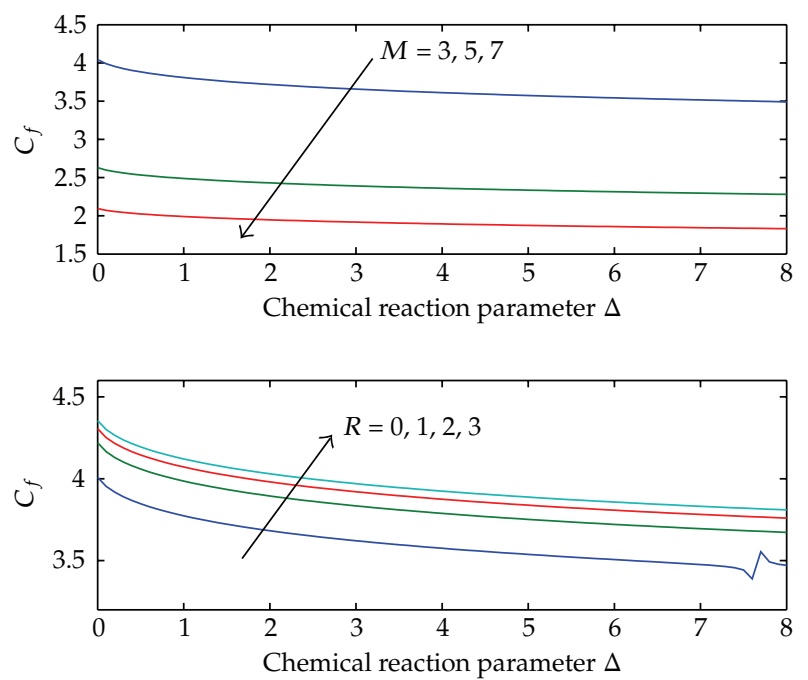

(a)
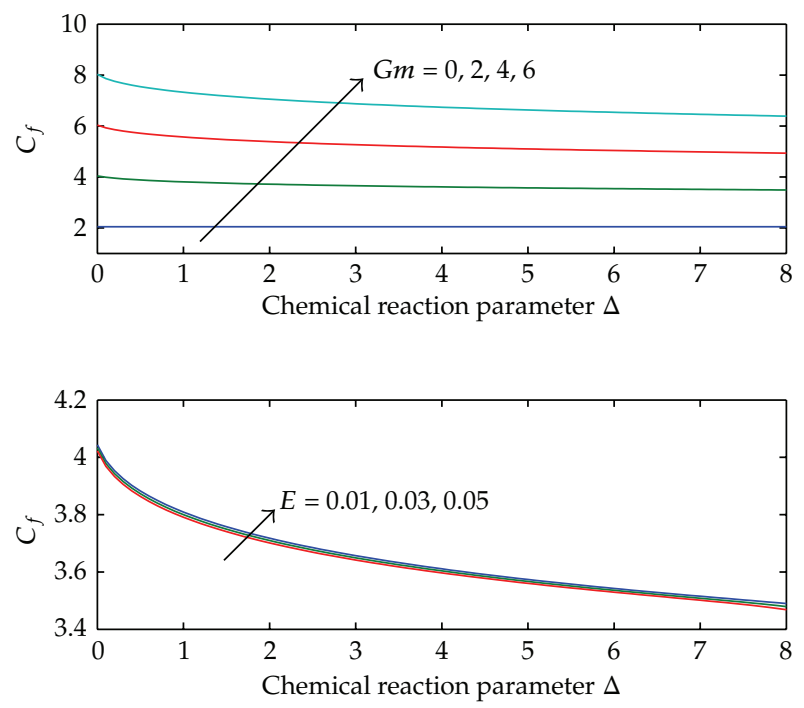

(b)

Figure 14: Effect of $M, R, G m$, and $E$ on $C_{f}$ respect to $\Delta$, where, $S c=0.22, \operatorname{Pr}=0.71, Q=0.5, \alpha=0.1$, $\mathrm{Gr}=2$, and $\beta=2$.

$y$ are presented in Figures 9 and 10, respectively. It is shown that the velocity $u$ increases with an increasing of $G m$ and $G r$ but the angular velocity decreases. In fact the increase in the value of $G m$ and $G r$ tends to increase the thermal and mass buoyancy effect. This gives rise to an increase in the induced flow. The effect of viscosity ratio $\alpha$ on the velocity angular velocity and temperature profiles across the boundary layer is presented in Figure 11. The numerical results show that the velocity distribution is lower for Newtonian fluid $(\alpha=0)$ with the fixed flow and material parameters, as compared with a polar fluid when the viscosity ratio is less than 0.5 . In addition, the angular velocity is decreased as $\alpha$-parameter 

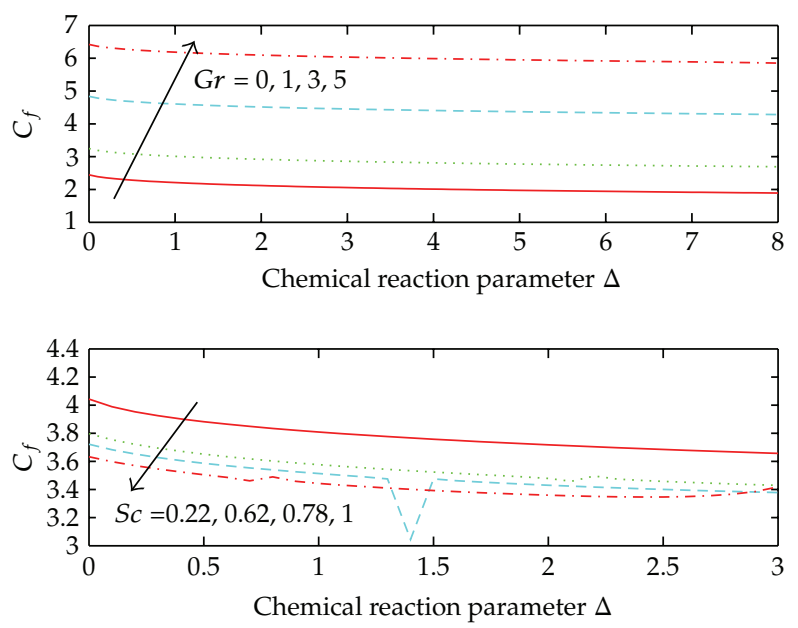

(a)
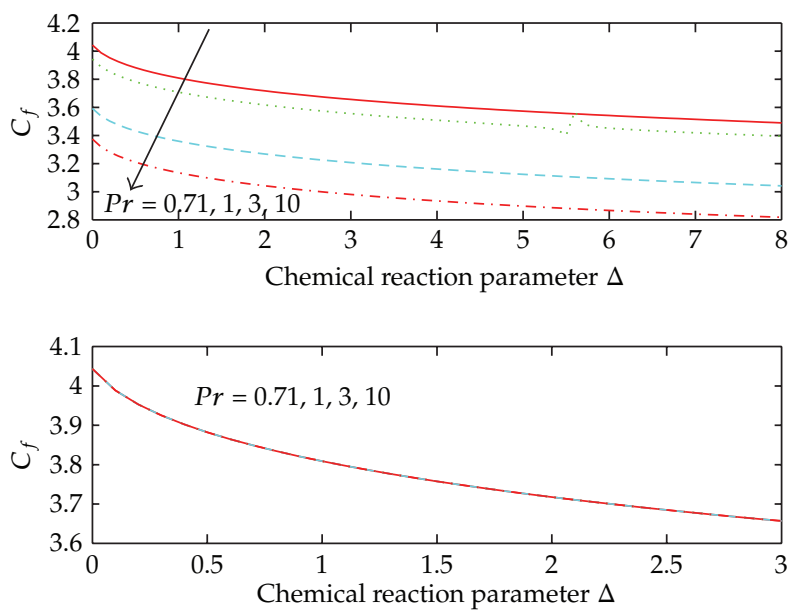

(b)

Figure 15: Effect of $G r, S c, \operatorname{Pr}$, and $k$ on $C_{f}$ respect to $\Delta$, where, $S_{c}=0.22, \Delta=0.5, \operatorname{Pr}=0.71, \alpha=0.1$, $G r=2$, and $\beta=2$.

increases. However, the distribution of angular velocity across the boundary layer does not show consistent variations with increment of $\alpha$-parameter. Also, it appears that there is a slight change in temperature with varies values of viscosity ratio $\alpha$. Representative velocity and angular velocity for various values of $\beta$ are illustrated in Figure 12. It can be seen that an increase in the polar parameter $\beta$ leads to a decrease in $u$ but an increase in $\omega$. From Figure 13 , we may note that the velocity $u$ and temperature $\theta$ change slightly to the increase of the viscous dissipation $E$ but the angular velocity $\omega$ increases with an increase of $E$.

The skin friction $\left(C_{f}\right)$, the wall heat transfer $(N u)$, and the wall mass transfer $(S h)$ coefficients are plotted in Figures 14-18, respectively. In Figures 14 and 15, the influences of $M, R, G m, E, G r, S c$, Pr, and $K$ on skin friction coefficient are plotted against chemical reaction parameter $\Delta$, respectively. Figure 16 displays the effects of $G m, E, M$, and $R$ on skin friction 

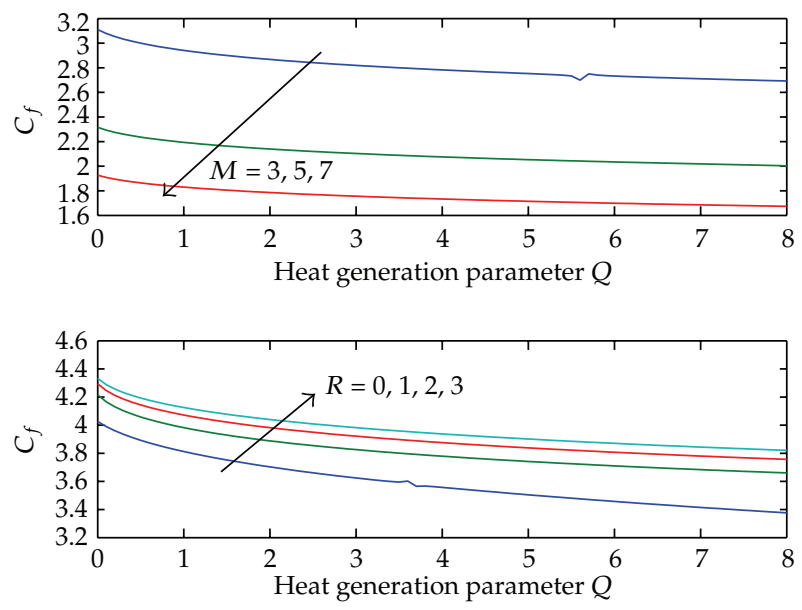

(a)
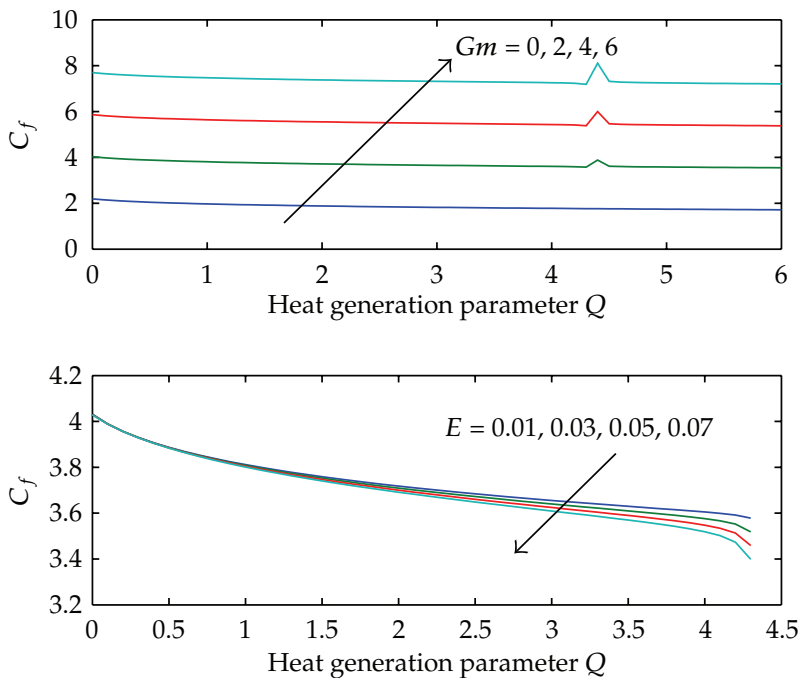

(b)

Figure 16: Effect of $G m, E, M$, and $R$ on $C_{f}$ respect to $Q$, where, $S c=0.22, \Delta=0.5, \operatorname{Pr}=0.71, \alpha=0.1$, $G r=2$, and $\beta=2$.

coefficient and plotted against heat generation parameter $Q$, respectively. From Figure 14, it is seen that $C_{f}$ increases with an increasing of $M, R$, and $G m$ but decreases with the increased values of $E$. From Figure 15, it appears that the skin friction $\left(C_{f}\right)$ increases with an increasing of $G r$ and $P r$ and decreases with the increased values of $S c$ but there is no sensitive influence with the various values of $k$. Also, It appears that from Figure 16 that $C_{f}$ increases with an increase of $R$ and $G m$ but decreases with the increasing values of $M$ and $E$. It is observed that the wall slope of the velocity decreases against the chemical reaction parameter $\Delta$ and heat generation parameter $Q$ as magnetic field $M$ and $E$ but increases with various values of solutal Grashof number $G m$ and the radiation $R$. Figure 17 displays the influence of heat generation parameter $Q$ on Nusselt number $N u$ (wall heat transfer coefficient) with influence of $\operatorname{Pr}, M$, and $R$. It is observed that the wall heat transfer coefficient increases as 

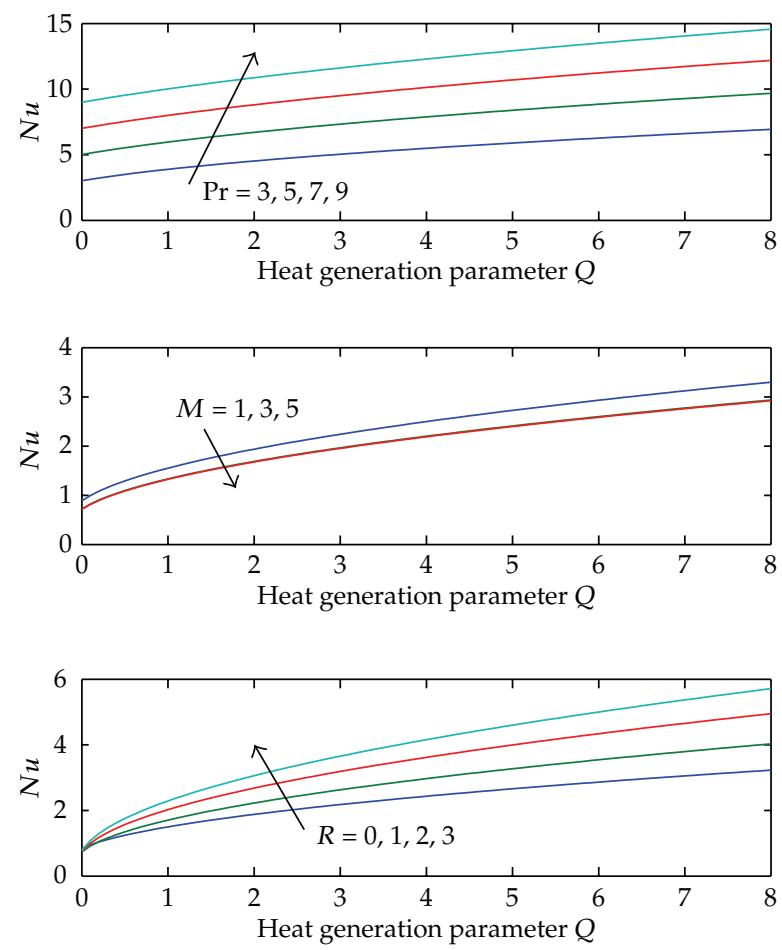

Figure 17: Effect of $\operatorname{Pr}, M$, and $R$ on wall heat transfer $N u$ respect to $Q$, where, $K=0.5, \operatorname{Pr}=0.71$, $\alpha=R=0.1, S c=0.22, G m=G r=M=2, E=0.01$, and $\beta=2$.

heat generation parameter is increased; also, with an increasing of $\mathrm{Pr}$ and $R$, the wall heat transfer coefficient increases but decreases with an increasing of the magnetic field $M$.

The Sherwood number Sh (wall mass transfer coefficient) is plotted in Figure 18 respect to chemical reaction $\Delta$ with influence of $S c$. It is observed that $S h$ increases with an increasing of $\Delta$ and Sc.

Finally, it is clear from Figures $2-13$ that the profiles of velocity $u$ increase with spanwise coordinate $y$ until it attains a maximum value, after which it decreases and approaches zero at the boundary. Also, it appears that the angular velocity $\omega$ starts from minimum negative value, after which it increases and tends to zero with an increasing of $y$; the negative values of the angular velocity indicate that the angular velocity of substructures in the polar fluid is clockwise, but the magnitude of the temperature $\theta$ and concentration $\phi$ start from their maximum value (unity) at the plate and then decays to approach zero asymptotically.

\section{Conclusion}

The work considered here provides an analysis of a two-dimensional steady radiative heat and mass transfer flow of an incompressible, laminar, and electrically conductive viscous 


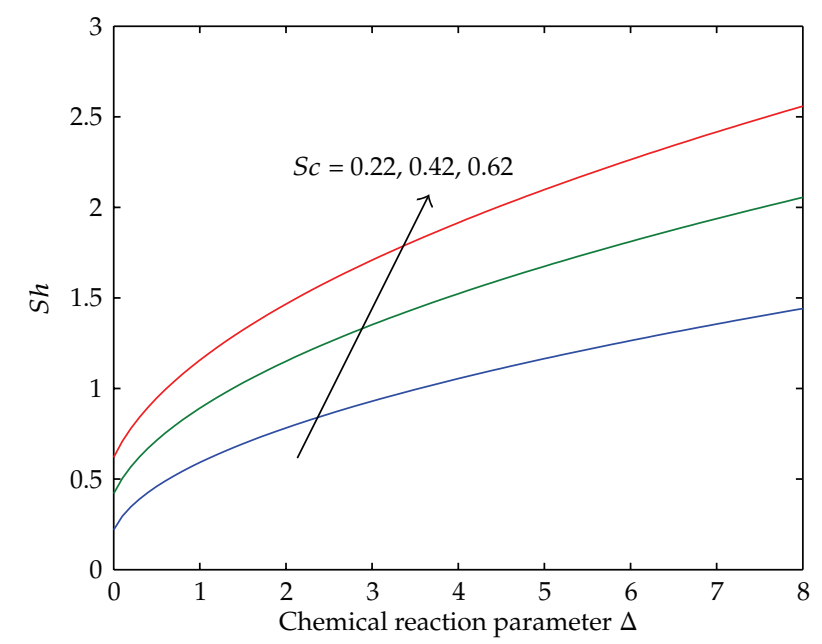

Figure 18: Effect of $S c$ on wall mass transfer $S h$ respect to $\Delta$, where, $K=0.5, \operatorname{Pr}=0.71, \alpha=R=0.1$, $G m=G r=M=2, Q=0.5, E=0.01$, and $\beta=2$.

dissipative polar fluid flow through a porous medium, occupying a semi-infinite region of the space bounded by an infinite vertical porous plate in the presence of a uniform transverse magnetic field, chemical reaction of the first order, and internal heat generation. Approximate solutions to the coupled non-linear equations governing the flow are derived and expression for the velocity, angular velocity, temperature, concentration, the rate of heat and mass transfer, and the skin-friction are derived. Results are presented graphically to illustrate the variation of velocity, angular velocity, temperature, concentration, skin friction, Nusselt number, and Sherwood number with various parameters. In this study, the following conclusions are set out:

(1) it appears that with an increasing of $R, K, G m, \alpha$, and $G r$, the velocity $u$ is increased and decreased with an increasing of $\Delta, S c, \operatorname{Pr}, Q, \beta$, and $M$ but there is a slight change with various values of $E$;

(2) it is concluded that the angular velocity $\omega$ increases with an increasing of $\Delta, S c, \mathrm{Pr}$, $\beta, E$ and $Q$ but decreases with an increasing of $R, K, M, G m, \alpha$, and $G r$;

(3) it is obvious that the temperature $\theta$ increases with an increasing of $R$ but decreases with an increasing of $\operatorname{Pr}$ and $Q$; there is a slight change with various values of $M$, $E$, and $\alpha$;

(4) it is clear that the concentration $\phi$ decreases with an increasing of $\Delta$ and $S c$;

(5) with various values of $R, G r$, and $G m$, the skin friction $C_{f}$ increases and decreases with an increasing of $M, \operatorname{Pr}, S c$, and $E$ with variation of $\Delta$ and $Q$;

(6) it is shown that Nusselt number increases with an increasing of Pr and $R$ but decreases with an increasing of $M$ with various values of $Q$;

(7) finally, it is displayed that Sherwood number $S h$ increases with an increasing of $S c$ with various values of $\Delta$. 


\section{Appendix}

The exponential indices in (3.8) are defined by

$$
\begin{aligned}
& R_{3,4}=\frac{-3 \operatorname{Pr} \mp \sqrt{9 \operatorname{Pr}^{2}-4(3+4 R)(3 \operatorname{Pr} Q)}}{2(3+4 R)}, \\
& R_{5,6}=\frac{-1 \mp \sqrt{1+(4(1+\alpha)(1+\alpha+K M)) / K}}{2(1+\alpha)}, \\
& A_{1}=\frac{-G r}{(1+\alpha)\left(R_{3}-R_{5}\right)\left(R_{3}-R_{6}\right)}, \quad A_{2}=\frac{-G m}{(1+\alpha)\left(R_{1}-R_{5}\right)\left(R_{1}-R_{6}\right)}, \\
& A_{3}=\frac{2 \alpha \beta}{(1+\alpha)\left(\beta+R_{5}\right)\left(\beta+R_{6}\right)}, \quad A_{4}=\frac{-3 \operatorname{PrC}_{2}^{2}\left(K^{-1}+R_{5}^{2}\right)}{4 R_{5}^{2}(3+4 R)+6 \operatorname{Pr} R_{5}+3 \operatorname{Pr} Q}, \\
& A_{5}=\frac{-3 \operatorname{Pr} A_{1}^{2}\left(K^{-1}+R_{3}^{2}\right)}{4 R_{3}^{2}(3+4 R)+6 \operatorname{Pr} R_{3}+3 \operatorname{Pr} Q}, \quad A_{6}=\frac{-3 \operatorname{Pr} A_{2}^{2}\left(K^{-1}+R_{1}^{2}\right)}{4 R_{1}^{2}(3+4 R)+6 \operatorname{Pr} R_{1}+3 \operatorname{Pr} Q}, \\
& A_{7}=\frac{-3 \operatorname{Pr} A_{3}^{2} C_{1}^{2}\left(K^{-1}+\beta^{2}\right)}{4 \beta^{2}(3+4 R)-6 \operatorname{Pr} \beta+3 \operatorname{Pr} Q}, \quad A_{8}=\frac{-6 \operatorname{Pr} A_{1} C_{2}\left(K^{-1}+R_{3} R_{5}\right)}{(3+4 R)\left(R_{3}+R_{5}\right)^{2}+3 \operatorname{Pr}\left(R_{3}+R_{5}\right)+3 \operatorname{Pr} Q}, \\
& A_{9}=\frac{-6 \operatorname{Pr} A_{2} C_{2}\left(K^{-1}+R_{1} R_{5}\right)}{(3+4 R)\left(R_{1}+R_{5}\right)^{2}+3 \operatorname{Pr}\left(R_{1}+R_{5}\right)+3 \operatorname{Pr} Q}, \quad A_{14}=\frac{-G r D_{1}}{(1+\alpha)\left(R_{3}-R_{5}\right)\left(R_{3}-R_{6}\right)}, \\
& A_{10}=\frac{-6 \operatorname{Pr} A_{3} C_{1} C_{2}\left(K^{-1}-\beta R_{5}\right)}{(3+4 R)\left(R_{5}-\beta\right)^{2}+3 \operatorname{Pr}\left(R_{5}-\beta\right)+3 \operatorname{Pr} Q}, \quad A_{15}=\frac{-G r A_{4}}{R_{5}(1+\alpha)\left(2 R_{5}-R_{6}\right)}, \\
& A_{11}=\frac{-6 \operatorname{Pr} A_{1} A_{2}\left(K^{-1}+R_{1} R_{3}\right)}{(3+4 R)\left(R_{1}+R_{3}\right)^{2}+3 \operatorname{Pr}\left(R_{1}+R_{3}\right)+3 \operatorname{Pr} Q}, \quad A_{16}=\frac{-G r A_{5}}{(1+\alpha)\left(2 R_{3}-R_{5}\right)\left(2 R_{3}-R_{6}\right)}, \\
& A_{12}=\frac{-6 \operatorname{Pr} A_{1} A_{3} C_{1}\left(K^{-1}-\beta R_{3}\right)}{(3+4 R)\left(R_{3}-\beta\right)^{2}+3 \operatorname{Pr}\left(R_{3}-\beta\right)+3 \operatorname{Pr} Q}, \quad A_{17}=\frac{-G r A_{6}}{(1+\alpha)\left(2 R_{1}-R_{5}\right)\left(2 R_{1}-R_{6}\right)}, \\
& A_{13}=\frac{-6 \operatorname{Pr} A_{2} A_{3} C_{1}\left(K^{-1}-\beta R_{1}\right)}{(3+4 R)\left(R_{1}-\beta\right)^{2}+3 \operatorname{Pr}\left(R_{1}-\beta\right)+3 \operatorname{PrQ} Q}, \quad A_{18}=\frac{-G r A_{7}}{(1+\alpha)\left(2 \beta+R_{5}\right)\left(2 \beta+R_{6}\right)}, \\
& A_{19}=\frac{-G r A_{8}}{R_{3}(1+\alpha)\left(R_{3}+R_{5}-R_{6}\right)}, \quad A_{20}=\frac{-G r A_{9}}{R_{1}(1+\alpha)\left(R_{1}+R_{5}-R_{6}\right)}, \\
& A_{21}=\frac{-G r A_{10}}{\beta(1+\alpha)\left(R_{5}-\beta-R_{6}\right)}, \quad A_{22}=\frac{-G r A_{11}}{(1+\alpha)\left(R_{1}+R_{3}-R_{5}\right)\left(R_{1}+R_{3}-R_{6}\right)},
\end{aligned}
$$




$$
\begin{aligned}
& A_{23}=\frac{-G r A_{12}}{(1+\alpha)\left(R_{3}-\beta-R_{5}\right)\left(R_{3}-\beta-R_{6}\right)}, \quad A_{24}=\frac{-G r A_{13}}{(1+\alpha)\left(R_{1}-\beta-R_{5}\right)\left(R_{1}-\beta-R_{6}\right)} \\
& A_{25}=A_{3}, \\
& C_{1}=\frac{A_{1}\left(R_{5}^{2}-R_{3}^{2}\right)+A_{2}\left(R_{5}^{2}-R_{1}^{2}\right)}{A_{3}\left(\beta^{2}-R_{5}^{2}\right)-\beta}, \quad C_{2}=\frac{A_{3}\left(R_{3}^{2} A_{1}+R_{1}^{2} A_{2}\right)+\left(A_{1}+A_{2}\right)\left(\beta-A_{3} \beta^{2}\right)}{A_{3}\left(\beta^{2}-R_{5}^{2}\right)-\beta} \\
& C_{3}=\frac{H_{2}-R_{5}^{2} H_{1}}{A_{25}\left(\beta^{2}-R_{5}^{2}\right)-\beta}, \quad C_{4}=\frac{H_{1}\left(\beta^{2} A_{25}-\beta\right)-H_{2} A_{25}}{A_{25}\left(\beta^{2}-R_{5}^{2}\right)-\beta}, \\
& D_{1}=-\sum_{i=4}^{13} A_{i}, \quad H_{1}=-\sum_{j=14}^{24} A_{j}, \quad \\
& H_{2}=-\left[R_{3}^{2} A_{14}+4 R_{5}^{2} A_{15}+4 R_{3}^{2} A_{16}+4 R_{1}^{2} A_{17}+4 \beta^{2} A_{18}+\left(R_{3}+R_{5}\right)^{2} A_{19}+\left(R_{1}+R_{5}\right)^{2} A_{20}\right. \\
& \left.\quad+\left(R_{5}-\beta\right)^{2} A_{21}+\left(R_{1}+R_{3}\right)^{2} A_{22}+\left(R_{5}-\beta\right)^{2} A_{23}+\left(R_{1}-\beta\right)^{2} A_{24}\right] .
\end{aligned}
$$

\section{Nomenclature}

$B_{0}$ : The magnetic induction Tesla

$C^{*}: \quad$ The species concentration $\mathrm{mol} \cdot \mathrm{m}^{-3}$

$C_{w}^{*}: \quad$ The surface concentration $\mathrm{mol} \cdot \mathrm{m}^{-3}$

$C_{\infty}^{*}$ : The species concentration far from the surface $\mathrm{mol} \cdot \mathrm{m}^{-3}$

$C_{a}, C_{d}$ : The coefficients of couple stress viscosities

$C_{f}$ : The skin-friction coefficient

$C_{m}$ : The couple stress coefficient

$c_{p}$ : The specific heat at constant pressure $\mathrm{J} \cdot \mathrm{kg}^{-1} \cdot \mathrm{K}^{-1}$

$D^{*}: \quad$ The chemical molecular diffusivity $\mathrm{m}^{2} \cdot \mathrm{s}^{-1}$

E: $\quad$ The Eckert number

Gm: The solutal Grashof number

Gr: $\quad$ Grashof number

g: $\quad$ The acceleration due to the gravity $\mathrm{m} \cdot \mathrm{s}^{-2}$

I: The scalar constants of dimension equal to that of the moment of inertia of unit mass $\mathrm{m}^{2}$

$k$ : $\quad$ The radius of gyration of the polar fluid $\mathrm{m}$

$K^{\prime}: \quad$ The permeability of the porous media $\mathrm{m}^{-1}$

$K^{*}: \quad$ The mean absorption coefficient $\mathrm{m}^{-1}$

K: $\quad$ The dimensionless permeability

$K_{1}: \quad$ The first-order chemical reaction rate $\mathrm{s}^{-1}$

M: $\quad$ The local magnetic field parameter

Nu: $\quad$ Nusselt number

Pr: $\quad$ The fluid Prandtl number

$Q_{0}: \quad$ The heat generation coefficient $\mathrm{W} \cdot \mathrm{m}^{-3} \cdot \mathrm{K}^{-1}$ 


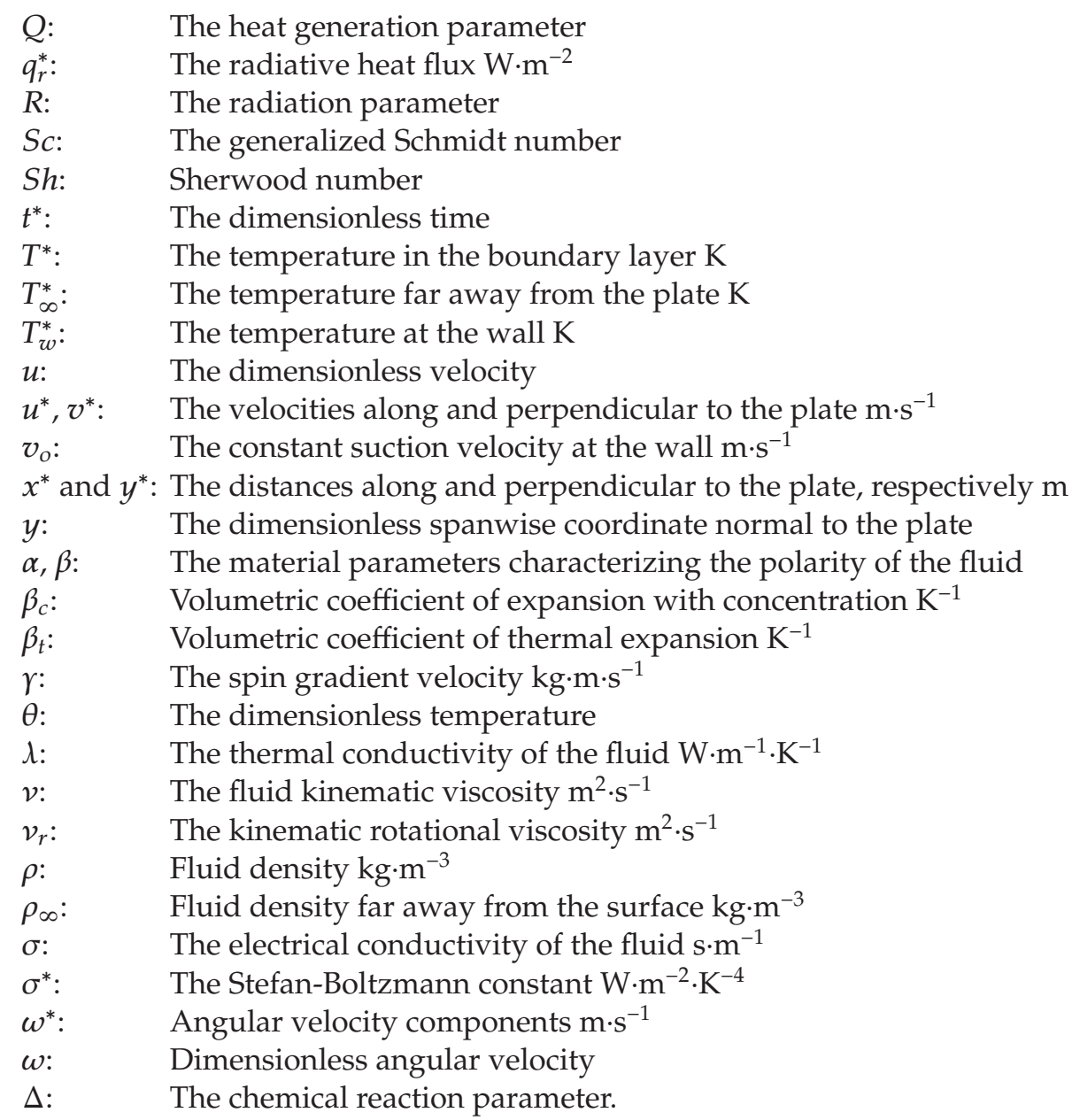

\section{References}

[1] U. N. Das, R. Deka, and V. M. Soundalgekar, “Effects of mass transfer on flow past an impulsively started infinite vertical plate with constant heat flux and chemical reaction," Forschung im Ingenieurwesen, vol. 60, no. 10, pp. 284-287, 1994.

[2] H. I. Andersson, O. R. Hansen, and B. Holmedal, "Diffusion of a chemically reactive species from a stretching sheet," International Journal of Heat and Mass Transfer, vol. 37, no. 4, pp. 659-664, 1994.

[3] S. P. Anjali Devi and R. Kandasamy, "Effects of chemical reaction, heat and mass transfer on laminar flow along a semi infinite horizontal plate," Warme- und Stoffubertragung Zeitschrift, vol. 35, no. 6, pp. 465-467, 1999.

[4] R. Muthucumaraswamy and P. Ganesan, "On impulsive motion of a vertical plate with heat flux and diffusion of chemically reactive species," Forschung im Ingenieurwesen, vol. 66, no. 1, pp. 17-23, 2000.

[5] R. Muthucumaraswamy and P. Ganesan, "First-order chemical reaction on flow past an impulsively started vertical plate with uniform heat and mass flux," Acta Mechanica, vol. 147, no. 1-4, pp. 45-57, 2001.

[6] R. Muthucumaraswamy, "Effects of suction on heat and mass transfer along a moving vertical surface in the presence of chemical reaction," Forschung im Ingenieurwesen, vol. 67, no. 3, pp. 129-132, 2002.

[7] R. Muthucumaraswamy, "Effects of a chemical reaction on a moving isothermal vertical surface with suction," Acta Mechanica, vol. 155, no. 1-2, pp. 65-70, 2002. 
[8] A. Y. Ghaly and M. A. Seddeek, "Chebyshev finite difference method for the effects of chemical reaction, heat and mass transfer on laminar flow along a semi infinite horizontal plate with temperature dependent viscosity," Chaos, Solitons and Fractals, vol. 19, no. 1, pp. 61-70, 2004.

[9] R. Kandasamy, K. Periasamy, and K. K. S. Prabhu, "Effects of chemical reaction, heat and mass transfer along a wedge with heat source and concentration in the presence of suction or injection," International Journal of Heat and Mass Transfer, vol. 48, no. 7, pp. 1388-1394, 2005.

[10] R. Kandasamy, K. Periasamy, and K. K. Sivagnana Prabhu, "Chemical reaction, heat and mass transfer on MHD flow over a vertical stretching surface with heat source and thermal stratification effects," International Journal of Heat and Mass Transfer, vol. 48, no. 21-22, pp. 4557-4561, 2005.

[11] R. A. Mohamed, I. A. Abbas, and S. M. Abo-Dahab, "Finite element analysis of hydromagnetic flow and heat transfer of a heat generation fluid over a surface embedded in a non-Darcian porous medium in the presence of chemical reaction," Communications in Nonlinear Science and Numerical Simulation, vol. 14, no. 4, pp. 1385-1395, 2009.

[12] K. Vajravelu and A. Hadjinicolaou, "Heat transfer in a viscous fluid over a stretching sheet with viscous dissipation and internal heat generation," International Communications in Heat and Mass Transfer, vol. 20, no. 3, pp. 417-430, 1993.

[13] A. J. Chamkha and I. Camille, "Effects of heat generation/absorption and thermophoresis on hydromagnetic flow with heat and mass transfer over a flat surface," International Journal of Numerical Methods for Heat and Fluid Flow, vol. 10, no. 4, pp. 432-448, 2000.

[14] A. J. Chamkha, "Effects of heat generation on g-jitter induced natural convection flow in a channel with isothermal or isoflux walls," Heat and Mass Transfer, vol. 39, no. 7, pp. 553-560, 2003.

[15] M. A. El-Hakiem, "Natural convection in a micropolar fluid with thermal dispersion and internal heat generation," International Communications in Heat and Mass Transfer, vol. 31, no. 8, pp. 1177-1186, 2004.

[16] S. Bagai, "Effect of variable viscosity on free convection over a non-isothermal axisymmetric body in a porous medium with internal heat generation," Acta Mechanica, vol. 169, no. 1-4, pp. 187-194, 2004.

[17] M. M. Molla, M. A. Hossain, and L. S. Yao, "Natural convection flow along a vertical wavy surface with uniform surface temperature in presence of heat generation/absorption," International Journal of Thermal Sciences, vol. 43, no. 2, pp. 157-163, 2004.

[18] F. M. Hady, R. A. Mohamed, and A. Mahdy, "MHD free convection flow along a vertical wavy surface with heat generation or absorption effect," International Communications in Heat and Mass Transfer, vol. 33, no. 10, pp. 1253-1263, 2006.

[19] M. M. Molla, M. A. Hossain, and M. C. Paul, "Natural convection flow from an isothermal horizontal circular cylinder in presence of heat generation," International Journal of Engineering Science, vol. 44, no. 13-14, pp. 949-958, 2006.

[20] A. J. Chamkha, A. F. Al-Mudhaf, and I. Pop, "Effect of heat generation or absorption on thermophoretic free convection boundary layer from a vertical flat plate embedded in a porous medium," International Communications in Heat and Mass Transfer, vol. 33, no. 9, pp. 1096-1102, 2006

[21] M. E. Ali, "The effect of lateral mass flux on the natural convection boundary layers induced by a heated vertical plate embedded in a saturated porous medium with internal heat generation," International Journal of Thermal Sciences, vol. 46, no. 2, pp. 157-163, 2007.

[22] P. M. Patil and P. S. Kulkarni, "Effects of chemical reaction on free convective flow of a polar fluid through a porous medium in the presence of internal heat generation," International Journal of Thermal Sciences, vol. 47, no. 8, pp. 1043-1054, 2008.

[23] V. M. Soundalgekar and H. S. Takhar, "MHD forced and free convection flow past a semi infinite plate," AIAA Journal, pp. 457-458, 1977.

[24] E. L. Aero, A. N. Bulygin, and E. V. Kuvshinskiio, “Asymmetric hydromechanics," Journal of Applied Mathematics and Mechanics, vol. 29, no. 2, pp. 333-346, 1965.

[25] N. van D'ep, "Equations of a fluid boundary layer with couple stresses," Journal of Applied Mathematics and Mechanics, vol. 32, no. 4, pp. 777-783, 1968.

[26] G. Łukaszewicz, Micropolar Fluids: Theory and Applications, Modeling and Simulation in Science, Engineering and Technology, Birkhäuser, Boston, Mass, USA, 1999.

[27] F. S. Ibrahim, I. A. Hassanien, and A. A. Bakr, “Unsteady magnetohydrodynamic micropolar fluid flow and heat transfer over a vertical porous plate through a porous medium in the presence of thermal and mass diffusion with a constant heat source," Canadian Journal of Physics, vol. 82, no. 10, pp. 775-790, 2004. 
[28] M. M. Rahman and M. A. Sattar, "Magnetohydrodynamic convective flow of a micropolar fluid past a continuously moving vertical porous plate in the presence of heat generation/absorption," Journal of Heat Transfer, vol. 128, no. 2, pp. 142-152, 2006.

[29] Y. J. Kim, "Unsteady MHD convection flow of polar fluids past a vertical moving porous plate in a porous medium," International Journal of Heat and Mass Transfer, vol. 44, no. 15, pp. 2791-2799, 2001.

[30] K. A. Helmy, "MHD unsteady free convection flow past a vertical porous plate," Zeitschrift für Angewandte Mathematik und Mechanik, vol. 78, no. 4, pp. 255-270, 1998.

[31] A. Ogulu, "The influence of radiation absorption on unsteady free convection and mass transfer flow of a polar fluid in the presence of a uniform magnetic field," International Journal of Heat and Mass Transfer, vol. 48, no. 23-24, pp. 5078-5080, 2005.

[32] S. P. Anjali Devi and R. Kandasamy, "Effects of chemical reaction, heat and mass transfer on MHD flow past a semi infinite plate," Zeitschrift fur Angewandte Mathematik und Mechanik, vol. 80, no. 10, pp. 697-701, 2000.

[33] H. S. Takhar, A. J. Chamkha, and G. Nath, "Flow and mass transfer on a stretching sheet with a magnetic field and chemically reactive species," International Journal of Engineering Science, vol. 38, no. 12, pp. 1303-1314, 2000.

[34] A. Raptis and C. Perdikis, "Viscous flow over a non-linearly stretching sheet in the presence of a chemical reaction and magnetic field," International Journal of Non-Linear Mechanics, vol. 41, no. 4, pp. 527-529, 2006.

[35] M. A. Seddeek, "Finite-element method for the effects of chemical reaction, variable viscosity, thermophoresis and heat generation/absorption on a boundary-layer hydromagnetic flow with heat and mass transfer over a heat surface," Acta Mechanica, vol. 177, no. 1-4, pp. 1-18, 2005.

[36] R. C. Sharma and K. D. Thakur, "On couple-stress fluid heated from below in porous medium in hydromagnetics," Czechoslovak Journal of Physics, vol. 50, no. 6, pp. 753-758, 2000.

[37] V. Sharma and S. Sharma, "Thermosolutal convection of micropolar fluids in hydromagnetics in porous medium," Indian Journal of Pure and Applied Mathematics, vol. 31, no. 10, pp. 1353-1367, 2000.

[38] Y. J. Kim, "Heat and mass transfer in MHD micropolar flow over a vertical moving porous plate in a porous medium," Transport in Porous Media, vol. 56, no. 1, pp. 17-37, 2004.

[39] A. Sunil, A. Sharma, P. K. Bharti, and R. G. Shandil, "Effect of rotation on a layer of micropolar ferromagnetic fluid heated from below saturating a porous medium," International Journal of Engineering Science, vol. 44, no. 11-12, pp. 683-698, 2006.

[40] V. M. Soundalgekar, "Free convection effects on stokes problem for a vertical plate," Journal of Heat Transfer, vol. 99, no. 3, pp. 499-501, 1977.

[41] A. Raptis, "Flow of a micropolar fluid past a continuously moving plate by the presence of radiation," International Journal of Heat and Mass Transfer, vol. 41, no. 18, pp. 2865-2866, 1998.

[42] Y. J. Kim and A. G. Fedorov, “Transient mixed radiative convection flow of a micropolar fluid past a moving, semi-infinite vertical porous plate," International Journal of Heat and Mass Transfer, vol. 46, no. 10, pp. 1751-1758, 2003.

[43] H. A. M. El-Arabawy, "Effect of suction/injection on the flow of a micropolar fluid past a continuously moving plate in the presence of radiation," International Journal of Heat and Mass Transfer, vol. 46, no. 8, pp. 1471-1477, 2003.

[44] M. A. Rahman and M. A. Sattar, "Transient convective flow of micropolar fluid past a continuously moving vertical porous plate in the presence of radiation," International Journal of Applied Mechanics and Engineering, vol. 12, no. 2, pp. 497-513, 2007.

[45] E. M. Abo-Eldahab and A. F. Ghonaim, "Radiation effect on heat transfer of a micropolar fluid through a porous medium," Applied Mathematics and Computation, vol. 169, no. 1, pp. 500-510, 2005.

[46] A. Ogulu, "On the oscillating plate-temperature flow of a polar fluid past a vertical porous plate in the presence of couple stresses and radiation," International Communications in Heat and Mass Transfer, vol. 32, no. 9, pp. 1231-1243, 2005.

[47] M. A. Rahman and T. Sultan, "Radiative heat transfer flow of micropolar fluid with variable heat flux in a porous medium," Nonlinear Analysis Modeling and Control, vol. 13, no. 1, pp. 71-87, 2008.

[48] R. A. Mohamed and S. M. Abo-Dahab, "Influence of chemical reaction and thermal radiation on the heat and mass transfer in MHD micropolar flow over a vertical moving porous plate in a porous medium with heat generation," International Journal of Thermal Sciences, vol. 48, pp. 1800-1813, 2009.

[49] R. Bayron Bird, W. E. Strwart, and E. N. Lightfoot, Transfer Phenomena, John Wiley and Sons, New York, NY, USA, 1992. 
[50] S. Whitakar, "Advances in theory of fluid motion in porous media," Industrial and Engineering Chemistry, vol. 61, no. 12, pp. 14-28, 1969.

[51] E. M. Sparrow and R. D. Cess, Radiation Heat Transfer, chapters 7, 10 and 19, Hemisphere, Washington, DC, USA, 1978. 


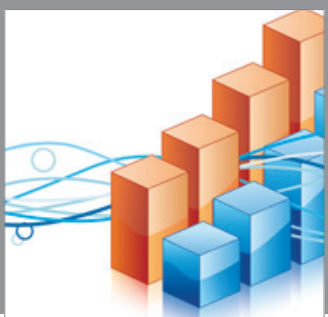

Advances in

Operations Research

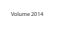

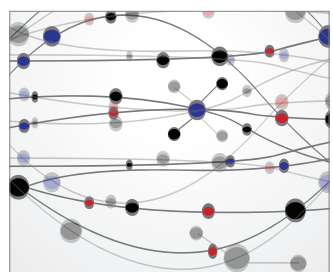

\section{The Scientific} World Journal
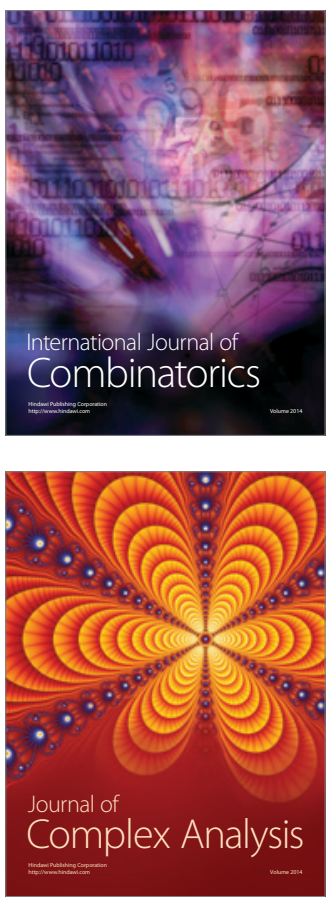

International Journal of

Mathematics and

Mathematical

Sciences
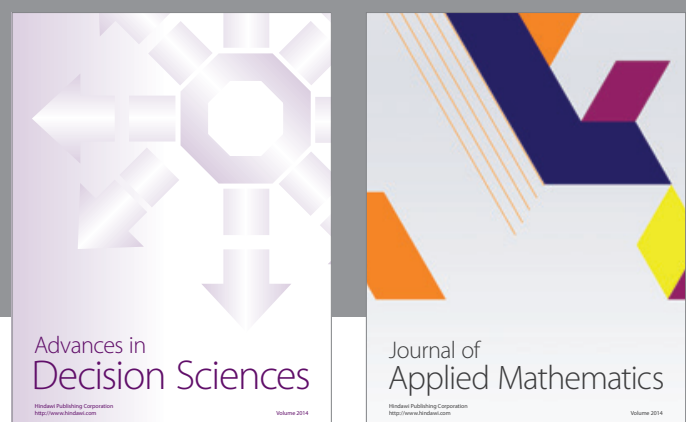

Journal of

Applied Mathematics
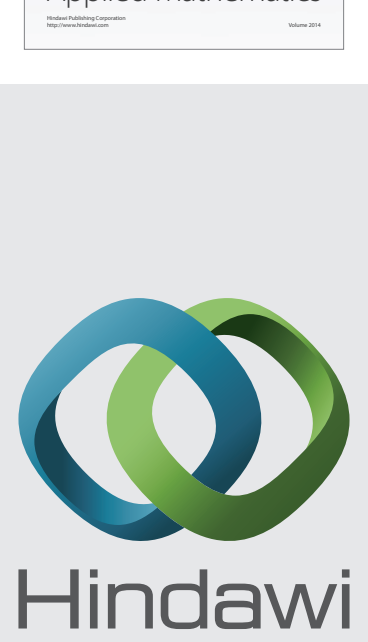

Submit your manuscripts at http://www.hindawi.com
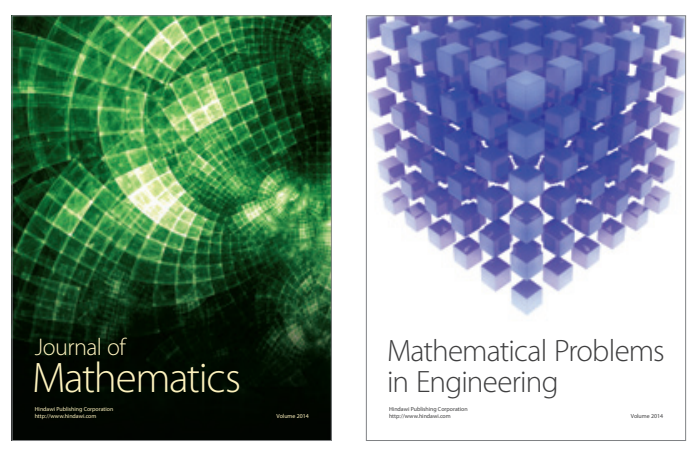

Mathematical Problems in Engineering
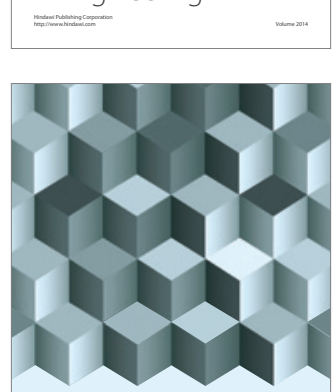

Journal of

Function Spaces
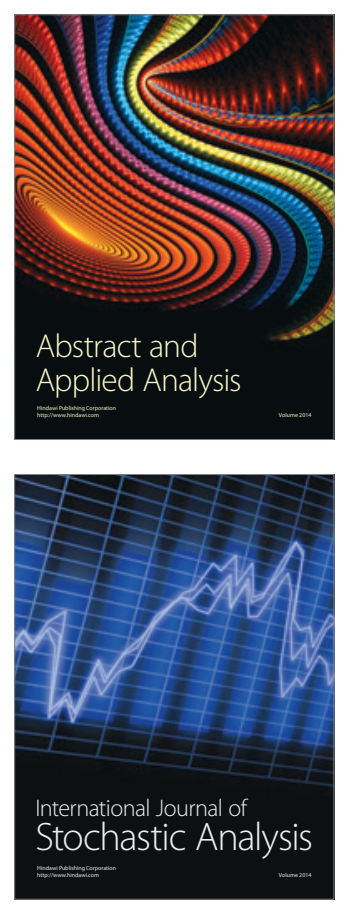

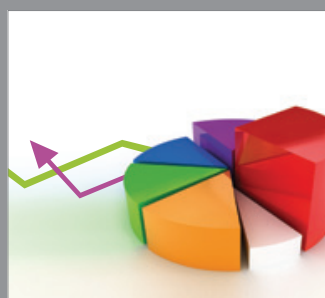

ournal of

Probability and Statistics

Promensencen
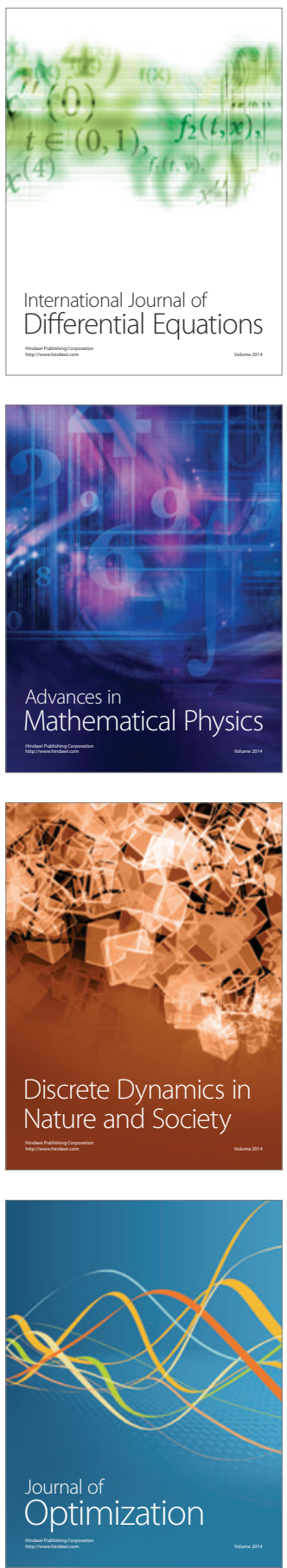